\title{
Management of advanced gastric cancer: An overview of major findings from meta-analysis
}

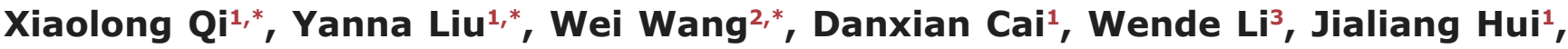 \\ Chuan Liu', Yanxia Zhao ${ }^{4}$ and Guoxin $\mathrm{Li}^{1}$ \\ ${ }^{1}$ Department of General Surgery, Nanfang Hospital, Southern Medical University, Guangzhou, China \\ 2 Department of Gastric and Pancreatic Surgery, Sun Yat-sen University Cancer Center, Guangzhou, China \\ ${ }^{3}$ Guangdong Laboratory Animal Monitoring Institute, Guangzhou, China \\ ${ }^{4}$ Cancer Center, Union Hospital, Tongji Medical College, Huazhong University of Science and Technology, Wuhan, China \\ * These authors contributed equally to this work \\ Correspondence to: Xiaolong Qi, email: qixiaolong@vip.163.com
}

Guoxin Li, email: gzliguoxin@163.com

Yanxia Zhao, email: sophia7781@126.com

Keywords: advanced gastric cancer, management, surgery, chemotherapy, meta-analysis

Received: June 30, $2016 \quad$ Accepted: August 26, 2016

Published: September 17, 2016

\section{ABSTRACT}

This study aims to provide an overview of different treatment for advanced gastric cancer. In the present study, we systematically reviewed the major findings from relevant meta-analyses. A total of 54 relevant papers were searched via the PubMed, Web of Science, and Google scholar databases. They were classified according to the mainstay treatment modalities such as surgery, chemotherapy and others. Primary outcomes including overall survival, response rate, disease-free survival, recurrence-free survival, progression-free survival, time-to-progression, time-to failure, recurrence and safety were summarized. The recommendations and uncertainties regarding the treatment of advanced gastric cancer were also proposed. It was suggested that laparoscopic gastrectomy was a safe and technical alternative to open gastrectomy. Besides, neoadjuvant chemotherapy and adjuvant chemotherapy were thought to benefit the survival over surgery alone. And it was demonstrated in the study that targeted therapy like anti-angiogenic and anti-HER2 agents but antiEGFR agent might have a significant survival benefit.

\section{INTRODUCTION}

In global, about 952,000 cases of gastric cancer (GC) are newly diagnosed every year. As the thirdleading cause of cancer related mortality, GC accounts for 841000 deaths in 2013 globally [1, 2]. GC is early detected more commonly in Japan and South Korea, probably due to active screening programs. In China, $\mathrm{GC}$ is the second diagnosed cancer, incidence of which comes to $67.9 \%$ [3]. Unfortunately, more than half of radically resected GC patients relapse locally or with distant metastases, or receive the diagnosis of GC when tumor is disseminated. Thus median survival rarely exceeds 12 months, and 5-year survival is less than $10 \%$ [4]. For locally advanced gastric cancer (AGC), surgery remains the only curative therapy. The use of laparoscopic gastrectomy (LG) in AGC remains controversial because of doubts about its efficacy and safety. Perioperative and adjuvant chemotherapy (AC), as well as chemoradiation are thought to bring benefits. For unresectable AGC with overt metastatic disease, palliative chemotherapy, targeted therapy and basic supportive care (BSC) are recommended by the National Comprehensive Cancer Network ( $\mathrm{NCCN}$ ) guideline and Japanese Gastric Cancer Association (JGCA) guideline [5]. In patients with good performance status, chemotherapy is better than BSC only [4]. Though positive effect has been achieved in recent years with second- and further lines of chemotherapy, as well as using HER2-targeting drugs and ramucirumab for AGC, many phase III trials regarding the use of other targeted 
Table 1: Findings of meta-analyses: An overview of included studies regarding LG vs. OG

\begin{tabular}{|c|c|c|c|c|c|}
\hline $\begin{array}{l}\text { First } \\
\text { Author }\end{array}$ & Journal (Year) & Comparisons & OS & $\begin{array}{l}\text { SR, DFS, RFS, } \\
\text { PFS, Recurrence, } \\
\text { TTF,TTP } \\
\end{array}$ & Major comments \\
\hline Lu C[12] & $\begin{array}{l}\text { Surg } \\
\text { Endosc(2015) }\end{array}$ & $\begin{array}{l}\text { LADG vs. } \\
\text { ODG }\end{array}$ & NA. & NA. & $\begin{array}{l}\text { D2 lymphadenectomy } \\
\text { performed laparoscopically } \\
\text { was as effective as an open } \\
\text { procedure in AGC. }\end{array}$ \\
\hline Quan Y[13] & $\begin{array}{l}\text { Gastric Cancer } \\
(2015)\end{array}$ & LG vs. OG & $\begin{array}{l}\text { OS:1-, 3-, } 5-y: \\
\text { statistically similar. }\end{array}$ & $\begin{array}{l}\text { DFS: 3-, 5-year: } \\
\text { statistically similar. } \\
\text { Recurrence: favor } \\
\text { LG. }\end{array}$ & $\begin{array}{l}\text { LG appeared comparable with } \\
\text { OG in short- and long-term } \\
\text { results. }\end{array}$ \\
\hline $\begin{array}{l}\text { Huang } \\
\text { YL[17] }\end{array}$ & $\begin{array}{l}\text { Int J Clin Exp } \\
\operatorname{Med}(2014)\end{array}$ & LAG vs. OG & $\begin{array}{l}\text { OS: } 3-y: \text { statistically } \\
\text { similar. }\end{array}$ & $\begin{array}{l}\text { Recurrence: } \\
\text { statistically similar. }\end{array}$ & $\begin{array}{l}\text { LAG with D2 lymph node } \\
\text { dissection was a feasible and } \\
\text { safe procedure for AGC. }\end{array}$ \\
\hline Zou ZH[26] & $\begin{array}{l}\text { World J } \\
\text { Gastroenterol } \\
(2014)\end{array}$ & $\begin{array}{l}\text { LGD2 vs. } \\
\text { OGD2 }\end{array}$ & $\begin{array}{l}\text { OS: } 3-, \quad 5-\quad \text { y: } \\
\text { statistically similar. }\end{array}$ & $\begin{array}{l}\text { DFS (3-, 5- year), } \\
\text { Recurrence/ } \\
\text { metastasis: } \\
\text { statistically similar. }\end{array}$ & $\begin{array}{l}\text { LGD2 might be safe and } \\
\text { effective, and offered some } \\
\text { advantages over OGD2 for } \\
\text { locally AGC. }\end{array}$ \\
\hline Chen K[27] & $\begin{array}{l}\text { World J Surg } \\
\text { Oncol (2013) }\end{array}$ & LG vs. OG & $\begin{array}{l}\text { OS } \quad(1-, \quad 3-, \quad 5- \\
\text { y), } \\
\text { statistically similar. }\end{array}$ & $\begin{array}{l}\text { DFS (3-, 5- year), } \\
\text { Recurrence: } \\
\text { statistically similar. }\end{array}$ & $\begin{array}{l}\text { LG was a safe technical } \\
\text { alternative to OG with a } \\
\text { lower complication rate } \\
\text { and enhanced postoperative } \\
\text { recovery. }\end{array}$ \\
\hline Ye LY[38] & $\begin{array}{l}\text { J Zhejiang Univ } \\
\text { Sci B (2013) }\end{array}$ & LAG vs. OG & NA. & $\begin{array}{l}\text { Recurrence: } \\
\text { statistically similar. }\end{array}$ & $\begin{array}{l}\text { LAG could be performed } \\
\text { safely for AGC with } \\
\text { adequate lymphadenectomy } \\
\text { and has several short-term } \\
\text { advantages. }\end{array}$ \\
\hline Qiu J[34] & $\begin{array}{l}\text { Surg Laparosc } \\
\text { Endosc Percutan } \\
\text { Tech }(2013)\end{array}$ & $\begin{array}{l}\text { LADG } \\
\text { ODG }\end{array}$ & $\begin{array}{l}\text { OS:3-y: statistically } \\
\text { similar. }\end{array}$ & $\begin{array}{l}\text { Recurrence: } \\
\text { statistically similar. }\end{array}$ & $\begin{array}{l}\text { The oncologic outcomes of } \\
\text { LADG for AGC patients } \\
\text { were comparable with open } \\
\text { approach. }\end{array}$ \\
\hline $\begin{array}{l}\text { Choi } \\
\text { YY[29] }\end{array}$ & $\begin{array}{l}\mathrm{J} \text { Surg Oncol } \\
(2013)\end{array}$ & LG vs. OG & Statistically similar. & $\begin{array}{l}\text { DFS: statistically } \\
\text { similar. }\end{array}$ & $\begin{array}{l}\text { There was no evidence that } \\
\text { LG was inferior to OG. }\end{array}$ \\
\hline $\begin{array}{l}\text { Martinez- } \\
\text { Ramos } \\
\text { D }[45]\end{array}$ & $\begin{array}{l}\text { Rev Esp Enferm } \\
\text { Dig (2011) }\end{array}$ & LG vs. OG & Statistically similar. & NA. & $\begin{array}{l}\text { LG was associated with a } \\
\text { longer operative time but } \\
\text { lower blood loss and shorter } \\
\text { postoperative hospital stay. }\end{array}$ \\
\hline
\end{tabular}

agents such as EGFR inhibitors or mTOR inhibitors have had negative results [6]. Thus, many questions on AGC treatment remain unresolved. In the present study, we systematically reviewed the major findings from all metaanalyses regarding the treatment of $\mathrm{AGC}$ and attempted to propose the evidence-based recommendations and uncertainties.

\section{RESULTS}

Overall, 239 papers were identified. Among them, 54 meta-analysis papers were finally included [7-60] (Figure 1). The characteristics of these included papers were shown in Supplementary Table S1. Their major findings were summarized according to the treatment modalities (Tables 1-18 and Supplementary Tables S2S21).

\section{Surgery}

\section{Laparoscopic gastrectomy (LG) vs. open gastrectomy (OG)}

Nine meta-analyses compared the outcomes of LG versus $\mathrm{OG}[12,13,17,26,27,29,34,38,45]$. There were 2,5 , and 3 meta-analyses comparing the 1-, 3-, 5-year survival, respectively [13, 17, 26, 27, 34]. Another two meta-analyses reported the overall survival (OS) without detailed time points [29, 45]. The meta-analyses all demonstrated that the OS was statistically similar between LG and OG [13, 17, 26, 27, 29, 34, 45]. Two of them did not report OS $[12,38]$. As for disease-free survival (DFS), three meta-analyses compared the 3- and 5-year DFS [17, $26,27]$ and another one reported DFS without detailed time point [29]. They all came to the conclusion that DFS was statistically similar between LG and OG [17, 26, 27, 29]. As for the recurrence, six of them found that it was statistically similar between the two groups $[13,17,26$, 
Table 2: Findings of meta-analyses: An overview of included studies regarding regarding NAC and AC

\begin{tabular}{|c|c|c|c|c|c|c|}
\hline First Author & Journal (Year) & Comparisons & OS & \begin{tabular}{|l|} 
SR, DFS, RFS, \\
PFS, \\
Recurrence, \\
TTF,TTP \\
\end{tabular} & Other & $\begin{array}{l}\text { Major } \\
\text { comments }\end{array}$ \\
\hline \multicolumn{7}{|c|}{ NAC vs. no chemotherapy before surgery } \\
\hline Xiao F[41] & $\begin{array}{l}\text { Chongqing } \\
\text { Medicine (2012) }\end{array}$ & $\begin{array}{l}\text { Surgery with vs. } \\
\text { without NAC }\end{array}$ & Favor NAC & NA. & $\begin{array}{l}\text { Resection rate: } \\
\text { favor NAC. } \\
\text { Peri-operative } \\
\text { mortality: } \\
\text { statistically } \\
\text { similar. }\end{array}$ & $\begin{array}{l}\text { NAC could } \\
\text { improve the } \\
\text { tumor resection } \\
\text { rate and the } \\
\text { survival rate in } \\
\text { AGC patients } \\
\text { without } \\
\text { increasing the } \\
\text { operative risk. } \\
\end{array}$ \\
\hline Li W[50] & $\begin{array}{l}\text { World J } \\
\text { Gastroenterol } \\
(2010)\end{array}$ & $\begin{array}{l}\text { Surgery with vs. } \\
\text { without NAC }\end{array}$ & Favor NAC & $\begin{array}{l}\text { PFS: 3-year: } \\
\text { favor NAC. }\end{array}$ & $\begin{array}{l}\text { Tumor down- } \\
\text { staging rate, R0 } \\
\text { resection rate: } \\
\text { favor NAC. } \\
\text { Peri- operative } \\
\text { mortality: } \\
\text { statistically } \\
\text { similar. } \\
\end{array}$ & $\begin{array}{l}\text { NAC could } \\
\text { improve tumor } \\
\text { stage and } \\
\text { survival rate of } \\
\text { patients with } \\
\text { AGC with a } \\
\text { rather good } \\
\text { safety. }\end{array}$ \\
\hline \multicolumn{7}{|c|}{ AC vs. surgery only } \\
\hline Sun J[36] & $\begin{array}{l}\text { BMC } \\
(2013)\end{array}$ & $\begin{array}{l}\text { (Include palliative } \\
\text { gastrectomy with } \\
\text { vs. without AC). }\end{array}$ & $\begin{array}{l}\text { Favor } \\
\text { palliative } \\
\text { gastrectomy } \\
\text { with AC. }\end{array}$ & NA. & NA. & \begin{tabular}{|l|} 
Palliative \\
gastrectomy \\
combined with \\
chemotherapy \\
maight improve \\
survival. \\
\end{tabular} \\
\hline Sun P[57] & Br J Surg (2009) & $\begin{array}{l}\text { Surgery with vs. } \\
\text { without AC. }\end{array}$ & $\begin{array}{l}\text { Favor } \\
\text { surgery with } \\
\text { AC. }\end{array}$ & NA. & NA. & $\begin{array}{l}\text { Postoperative } \\
\text { chemotherapy } \\
\text { could improve } \\
\text { OS after radical } \\
\text { surgery for } \\
\text { gastric cancer. }\end{array}$ \\
\hline
\end{tabular}
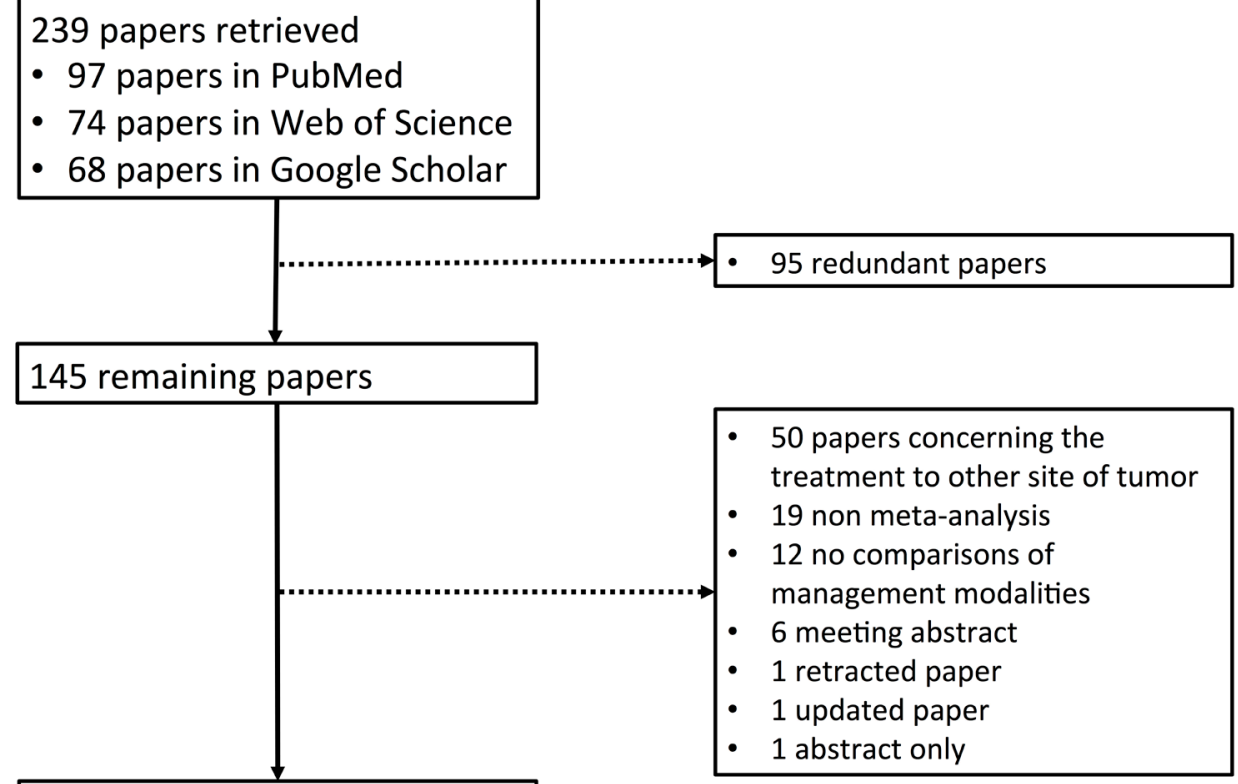

54 remaining papers

Figure 1: Flowchart of study inclusion 
Table 3: Findings of meta-analyses: An overview of included studies regarding Surgery with vs. without IPC

\begin{tabular}{|c|c|c|c|c|c|c|}
\hline First Author & $\begin{array}{l}\text { Journal } \\
\text { (Year) }\end{array}$ & Comparisons & OS & $\begin{array}{l}\text { SR, DFS, RFS, } \\
\text { PFS, } \\
\text { Recurrence, } \\
\text { TTF,TTP } \\
\end{array}$ & Other & $\begin{array}{l}\text { Major } \\
\text { comments }\end{array}$ \\
\hline Coccolini F[16] & $\begin{array}{l}\text { Eur J Surg } \\
\text { Oncol (2014) }\end{array}$ & $\begin{array}{l}\text { Surgery with } \\
\text { vs. without } \\
\text { IPC. }\end{array}$ & $\begin{array}{l}\text { OS: } \\
1-, 2-, 3- \\
\text { year: favor } \\
\text { surgery+IPC; } \\
\text { 5-year: } \\
\text { statistically } \\
\text { similar. }\end{array}$ & $\begin{array}{l} \\
\text { Overall } \\
\text { recurrence, } \\
\text { peritoneal } \\
\text { recurrence, } \\
\text { haematogenous } \\
\text { metastasis: favor } \\
\text { surgery + IPC. } \\
\text { Lymph-nodal } \\
\text { recurrence: } \\
\text { statistically } \\
\text { similar. }\end{array}$ & $\begin{array}{l}\text { Mortality: 1-, } \\
\text { 2-, 3-year: } \\
\text { favor surgery+ } \\
\text { IPC; 5-year: } \\
\text { statistically } \\
\text { similar; 2-, } \\
\text { 3-year in patients } \\
\text { with loco- } \\
\text { regional nodal } \\
\text { metastasis, 1, } \\
\text { 2-year in patients } \\
\text { with serosal } \\
\text { infiltration: favor } \\
\text { surgery+IPC. } \\
\text { Morbidity: higher } \\
\text { in surgery alone. }\end{array}$ & $\begin{array}{l}\text { IPC had } \\
\text { positive effect } \\
\text { on peritoneal } \\
\text { recurrence } \\
\text { and distant } \\
\text { metastasis. } \\
\text { Morbidity rate } \\
\text { is incremented } \\
\text { by IPC. Loco- } \\
\text { regional lymph- } \\
\text { nodes invasion } \\
\text { in patients } \\
\text { affected by } \\
\text { AGC was not a } \\
\text { contraindication } \\
\text { to IPC. }\end{array}$ \\
\hline Yan TD[58] & $\begin{array}{l}\text { Ann Surg } \\
\text { Oncol (2007) }\end{array}$ & $\begin{array}{l}\text { Surgery with } \\
\text { vs. without } \\
\text { IPC. }\end{array}$ & $\begin{array}{l}\text { Favor surgery } \\
\text { with HIIPC or } \\
\text { with HIIPC+ } \\
\text { EPIPC (but } \\
\text { statistically } \\
\text { similar } \\
\text { between } \\
\text { surgery with } \\
\text { NIIPC, EPIPC } \\
\text { or DPIPC } \\
\text { and surgery } \\
\text { without IPC). }\end{array}$ & $\begin{array}{l}\text { Peritoneal } \\
\text { recurrence } \\
\text { (surgery with } \\
\text { HIIPC or NIIPC } \\
\text { vs. control): } \\
\text { statistically } \\
\text { similar. }\end{array}$ & $\begin{array}{l}\text { Perioperative } \\
\text { mortality: } \\
\text { statistically } \\
\text { similar. Risk of } \\
\text { intra-abdominal } \\
\text { abscess, } \\
\text { neutropenia: } \\
\text { higher in IPC+ } \\
\text { surgery. }\end{array}$ & $\begin{array}{l}\text { HIIPC with or } \\
\text { without EPIPC } \\
\text { after resection } \\
\text { of AGC } \\
\text { improved the } \\
\text { overall survival. } \\
\text { However, } \\
\text { increased risk of } \\
\text { intra-abdominal } \\
\text { abscess and } \\
\text { neutropenia } \\
\text { were also } \\
\text { demonstrated. }\end{array}$ \\
\hline
\end{tabular}

$27,34,38]$ while the other three did not report relevant data. Although LG required longer operative time [17, $26,27,34,38,45]$, it offered some advantages over OG with lower blood loss [13, 17, 26, 27, 34, 38, 45], shorter hospitalization $[13,17,26,27,34,38,45]$ and quick recovery $[13,17,26,27,38]$.

Besides, three of them demonstrated that LG had fewer postoperative complications $[13,17,26]$, but another two did not show significant difference between the two groups $[34,38]$. In addition, LG was comparable to OG that the number of harvested lymph nodes was statistically similar between two groups $[12,13,17,26$, $27,34,38,45]$.

Only non-randomized controlled trial (RCT) studies were included in the meta-analyses by Lu $(n=8)$ and Qiu $(n=7)$. The meta-analyses by Huang did not report type of the included studies. In the meta-analyses by MartinezRamos, Choi, Ye, Zou and Quan, there was only one RCT regarding LG versus OG while Chen's meta-analysis had a larger number of RCTs.

The meta-analyses by Quan had the largest number of included studies $(n=26)$ followed by the meta-analyses by Chen $(n=15)$ and Zou $(n=14)$ (Supplementary Table S5). By comparison, the number of included studies was less than 20 in 8 other meta-analyses.
Given its superiority in the quantity of RCT studies, the results of the meta-analysis by Chen might be more reliable. In details, LG was a safe and technical alternative to $\mathrm{OG}$ for $\mathrm{AGC}$ with a lower complication rate and enhanced postoperative recovery.

\section{Neoadjuvant chemotherapy (NAC) vs. no therapy before surgery}

Two meta-analyses compared the outcomes of surgery in combination with NAC versus no therapy before surgery. Both of them favored NAC in terms of OS $[41,50]$. One of them also favored NAC in terms of 3-year progression-free survival (PFS) [50] while the other did not reported PFS. Besides, both of the meta-analyses demonstrated that the resection rate was higher for NAC group than for control group while the perioperative mortality showed no statistically difference $[41,50]$. Additionally, one of them revealed that NAC had a significant down-staging effect on AGC [50].

Only RCT studies were included in the metaanalysis by Xiao $(n=18)$ and the meta-analysis by $\mathrm{Li}(n=$ 14) did not report the details of the included studies.

The meta-analysis by Xiao had a larger number of included studies than those by Li (18 versus 14) (Supplementary Table S6). Notably, there was an overlap of included studies between the two meta-analyses. All 
Table 4: Findings of meta-analyses: An overview of included studies regarding lymphadenectomy

\begin{tabular}{|c|c|c|c|c|c|c|}
\hline First Author & Journal (Year) & Comparisons & $\mathbf{O S}$ & \begin{tabular}{|l|} 
SR, DFS, RFS, \\
PFS, \\
Recurrence, \\
TTF,TTP \\
\end{tabular} & Other & $\begin{array}{l}\text { Major } \\
\text { comments }\end{array}$ \\
\hline \multicolumn{7}{|c|}{ D2 vs. D4 lymphadenectomy } \\
\hline Wang Z[53] & $\begin{array}{l}\text { World J } \\
\text { Gastroenterol } \\
(2010)\end{array}$ & $\begin{array}{l}\text { D4 vs. D2 } \\
\text { lymphadenectomy }\end{array}$ & $\begin{array}{l}\text { OS: } \\
5-y: \\
\text { statistically } \\
\text { similar }\end{array}$ & NA. & $\begin{array}{l}\text { Postoperative } \\
\text { morbidity } \\
\text { and mortality: } \\
\text { statistically } \\
\text { similar; Operation } \\
\text { time, blood loss: } \\
\text { favor D2 group. }\end{array}$ & $\begin{array}{l}\text { D4 could be } \\
\text { performed } \\
\text { as safely as } \\
\text { standard D2 } \\
\text { dissection } \\
\text { without } \\
\text { increasing } \\
\text { post-operative } \\
\text { mortality but } \\
\text { failed to benefit } \\
\text { OS. }\end{array}$ \\
\hline Zhang YL[54] & $\begin{array}{l}\text { Chin J Gen } \\
\text { Surg (2010) }\end{array}$ & $\begin{array}{l}\text { D2 vs. D4 } \\
\text { lymphadenectomy }\end{array}$ & NA. & $\begin{array}{l}\text { SR: 5-y: } \\
\text { Statistically } \\
\text { similar; } \\
\text { Recurrence: } \\
\text { 5-y: } \\
\text { statistically } \\
\text { similar. }\end{array}$ & $\begin{array}{l}\text { Postoperative } \\
\text { mortality and } \\
\text { complications: } \\
\text { statistically } \\
\text { similar. }\end{array}$ & $\begin{array}{l}\text { The efficacy and } \\
\text { safety of D2 and } \\
\text { D4 lymph node } \\
\text { dissection for } \\
\text { AGC patients } \\
\text { was significantly } \\
\text { similar but } \\
\text { have yet to } \\
\text { be accurately } \\
\text { analysed. }\end{array}$ \\
\hline \multicolumn{7}{|c|}{ D2 vs. D3 lymphadenectomy } \\
\hline Zhang L[48] & $\begin{array}{l}\text { Chin Gen Prac } \\
(2011)\end{array}$ & $\begin{array}{l}\text { D2 vs. D3 } \\
\text { lymphadenectomy }\end{array}$ & NA. & $\begin{array}{l}\text { SR: 5-y: } \\
\text { statistically } \\
\text { similar }\end{array}$ & $\begin{array}{l}\text { Postoperative } \\
\text { mortality, } \\
\text { anastomotic } \\
\text { leakage, } \\
\text { pneumonia: } \\
\text { statistically } \\
\text { similar; Pancreatic } \\
\text { leakage, operative } \\
\text { time, blood } \\
\text { loss: favor D2 } \\
\text { lymphadenectomy. }\end{array}$ & $\begin{array}{l}\text { Further } \\
\text { rigorously } \\
\text { controlled } \\
\text { trials are } \\
\text { required before } \\
\text { conclusions on } \\
\text { which procedure } \\
\text { was more } \\
\text { favorable could } \\
\text { be made. }\end{array}$ \\
\hline
\end{tabular}

studies that were included in the meta-analysis by Li were also covered by the meta-analysis by Xiao.

Given its superiority in the quantity of RCT studies, the results of the meta-analysis by Xiao might be reliable. In details, NAC could improve the tumor resection rate and the survival rate in AGC patients without increasing the operative risk. Besides, perioperative mortality in two groups was statistically similar.

\section{Surgery with vs. without AC}

Two meta-analyses compared the outcomes of surgery combined with AC versus surgery without AC. Both of them favored surgery combine with AC. Other relevant outcomes were not evaluated in both metaanalyses [36, 57].

Only RCT studies were included in meta-analysis by Sun P while only non RCTs were included in Sun J's article.

The meta-analysis by Sun $\mathrm{P}$ had the larger number of included studies $(n=12)$ compared to the meta-analysis by Sun J ( $n=3)$ (Supplementary Table S7). Notably, there was an overlap of included studies between them.

Given its superiority in the quantity of RCT studies, the results of the meta-analysis by Sun P might be more reliable. In details, postoperative chemotherapy could improve OS after radical surgery for gastric cancer.

\section{Surgery with vs. without intraperitoneal chemotherapy (IPC)}

Two meta-analyses compared the outcomes of surgery combined with IPC versus surgery without IPC $[16,58]$. One of the meta-analyses revealed IPC significantly improve 1-, 2-, 3-year OS but not 5-year OS [16]. Another meta-analysis which also focused on adjuvant IPC demonstrated that hyperthermic intraoperative intraperitoneal chemotherapy (HIIPC) with or without early postoperative intraperitoneal chemotherapy (EPIPC) after gastrectomy was associated with improved OS; however, OS was statistically similar between surgery with normothermic intraoperative intraperitoneal chemotherapy (NIIPC), EPIC or delayed postoperative intraperitoneal chemotherapy (DPIPC) and 
Table 5: Findings of meta-analyses: An overview of included studies regarding other topics of "Surgery"

\begin{tabular}{|c|c|c|c|c|c|c|}
\hline First Author & $\begin{array}{l}\text { Journal } \\
\text { (Year) }\end{array}$ & Comparisons & OS & $\begin{array}{l}\text { SR, DFS, } \\
\text { RFS, } \\
\text { PFS, } \\
\text { Recurrence, } \\
\text { TTF, TTP }\end{array}$ & Other & Major comments \\
\hline \multicolumn{7}{|c|}{ Palliative gastrectomy vs. non-palliative gastrectomy } \\
\hline Sun J[36] & $\begin{array}{l}\text { BMC Cancer } \\
(2013)\end{array}$ & $\begin{array}{l}\text { Palliative } \\
\text { gastrectomy } \\
\text { vs. non- } \\
\text { palliative } \\
\text { gastrectomy. }\end{array}$ & $\begin{array}{l}\text { Favor palliative } \\
\text { gastrectomy } \\
\text { (especially } \\
\text { stage M1 GC) }\end{array}$ & NA. & NA. & $\begin{array}{l}\text { Palliative gastrectomy } \\
\text { for AGC might be } \\
\text { associated with longer } \\
\text { survival, especially for } \\
\text { patients with stage M1 } \\
\text { gastric cancer. }\end{array}$ \\
\hline \multicolumn{7}{|c|}{ Palliative gastrectomy with vs. without hepatic resection } \\
\hline Sun J[36] & $\begin{array}{l}\text { BMC Cancer } \\
(2013)\end{array}$ & $\begin{array}{l}\text { (Included } \\
\text { palliative } \\
\text { gastrectomy } \\
\text { with vs. } \\
\text { without } \\
\text { hepatic } \\
\text { resection). }\end{array}$ & $\begin{array}{l}\text { Favor } \\
\text { gastrectomy+ } \\
\text { hepatic } \\
\text { resection. }\end{array}$ & NA. & NA. & $\begin{array}{l}\text { Palliative gastrectomy } \\
\text { combined with hepatic } \\
\text { resection might } \\
\text { improve survival. }\end{array}$ \\
\hline \multicolumn{7}{|c|}{ Drain vs. no drain after gastrectomy } \\
\hline Liu HP[44] & $\begin{array}{l}\text { Dig } \\
(2011)\end{array}$ & $\begin{array}{l}\text { Drain vs. no } \\
\text { drain after } \\
\text { gastrectomy. }\end{array}$ & NA. & NA. & $\begin{array}{l}\text { Wound infection, } \\
\text { postoperative } \\
\text { pulmonary } \\
\text { infection, } \\
\text { intra-abdominal } \\
\text { abscess, } \\
\text { mortality, } \\
\text { number of } \\
\text { postoperative } \\
\text { days until } \\
\text { passing of flatus } \\
\text { and initiation } \\
\text { of soft diet: } \\
\text { statistically } \\
\text { similar. } \\
\text { Postoperative } \\
\text { complications, } \\
\text { hospital stay: } \\
\text { lower in the no- } \\
\text { drain group. }\end{array}$ & $\begin{array}{l}\text { lo use of } \\
\text { Avoiding the } \\
\text { abdominal drains } \\
\text { might reduce drain- } \\
\text { related complications } \\
\text { and shorten hospital } \\
\text { stay after gastrectomy. }\end{array}$ \\
\hline
\end{tabular}

surgery without IPC [58].

As for recurrence, one indicated that the overall recurrence and the peritoneal recurrence rates were improved by surgery combined with IPC [16]. However, another meta-analysis demonstrated that the peritoneal recurrence rates did not improved by surgery combined with IPC [58]. Besides, there was no significant difference in lymph-nodal recurrence rate; and the rate of haematogenous metastasis was improved by surgery combined with IPC [16].

As for mortality, one meta-analysis revealed that 1-, 2- and 3-year overall mortality was improved by IPC while 5-year overall mortality had no statistically difference between the two groups. Besides, 1- and 2-year mortality rate in patients with serosal infiltration as well as 2- and 3-year mortality rate in patients with loco-regional nodal metastasis were improved by the use of IPC. But morbidity rate is incremented by IPC [16].
Another meta-analysis showed that IPC did not improve the perioperative mortality compared to surgery alone. Besides, risk of intra-abdominal abscess and neutropenia were increased by IPC [58].

Only RCT studies, rather than non-RCTs, were included in the two meta-analyses.

The meta-analysis by Coccolini had the largest number of included studies $(n=20)$ followed by the meta-analysis by Yan $(n=13)$ (Supplementary Table S8). Notably, there was an overlap of included studies among them.

Given its superiority in the quantity of RCT studies, the results of the meta-analysis by Coccolini might be more reliable. In details, IPC had positive effect on overall and peritoneal recurrence and distant metastasis. Besides, loco-regional lymph-nodes invasion in patients affected by AGC was not a contraindication to IPC. 
Table 6: Findings of meta-analyses: An overview of included studies regarding chemotherapy vs. BSC

\begin{tabular}{|c|c|c|c|c|c|c|}
\hline $\begin{array}{l}\text { First } \\
\text { Author }\end{array}$ & $\begin{array}{l}\text { Journal } \\
\text { (Year) }\end{array}$ & Comparisons & OS & $\mathbf{R R}$ & $\begin{array}{c}\text { SR, DFS, } \\
\text { RFS, PFS, } \\
\text { Recurrence, } \\
\text { TTF,TTP }\end{array}$ & Major comments \\
\hline $\begin{array}{l}\text { Badiani } \\
\mathrm{B}[9]\end{array}$ & $\begin{array}{l}\text { World J } \\
\text { Clin Oncol } \\
(2015)\end{array}$ & $\begin{array}{l}6 \text { regimens of } \\
\text { chemotherapy } \\
\text { vs. BSC }\end{array}$ & $\begin{array}{l}\text { OS: paclitaxel with/ } \\
\text { without ramucirumab } \\
\text { vs. BSC: favor } \\
\text { chemotherapy; other } 4 \\
\text { vs. BSC: statistically } \\
\text { similar. }\end{array}$ & NA. & NA. & $\begin{array}{l}\text { Both paclitaxel } \\
\text { monotherapy and } \\
\text { ramucirumab }+ \\
\text { paclitaxel determined } \\
\text { a significant } \\
\text { prolongation in } \\
\text { survival as compared } \\
\text { with BSC. }\end{array}$ \\
\hline $\begin{array}{l}\text { Iacovelli } \\
\mathrm{R}[18]\end{array}$ & $\begin{array}{ll}\text { PLoS } & \text { One } \\
(2014) & \end{array}$ & $\begin{array}{l}\text { (Include } \\
\text { chemotherapy } \\
\text { vs. BSC) }\end{array}$ & $\begin{array}{l}\text { OS: patient } E C O G=0 \text { : } \\
\text { favor chemotherapy; } \\
\text { patient ECOG } \geq 1 \text { : favor } \\
\text { chemotherapy. }\end{array}$ & NA. & NA. & $\begin{array}{l}\text { Patients with } \\
\text { symptomatic } \\
\text { disease should not } \\
\text { be immediately } \\
\text { excluded by further } \\
\text { lines of therapy. }\end{array}$ \\
\hline $\begin{array}{l}\text { Kim } \\
\mathrm{HS}[31]\end{array}$ & $\begin{array}{l}\text { Ann Oncol } \\
(2013)\end{array}$ & $\begin{array}{l}\text { Second-line } \\
\text { chemotherapy } \\
\text { vs. BSC }\end{array}$ & $\begin{array}{l}\text { Favor Second-line } \\
\text { chemotherapy. }\end{array}$ & NA. & NA. & $\begin{array}{l}\text { This study } \\
\text { demonstrated } \\
\text { evidence to support } \\
\text { second-line } \\
\text { chemotherapy in } \\
\text { AGC. } \\
\end{array}$ \\
\hline $\begin{array}{l}\text { Wagner } \\
\mathrm{AD}[51]\end{array}$ & $\begin{array}{l}\text { Cochrane } \\
\text { Database } \\
\text { Syst Rev } \\
(2010)\end{array}$ & $\begin{array}{l}\text { (Include } \\
\text { chemotherapy } \\
\text { vs. BSC). }\end{array}$ & Favor chemotherapy & NA. & $\begin{array}{l}\text { TTP: favor } \\
\text { chemotherapy. }\end{array}$ & $\begin{array}{l}\text { Chemotherapy } \\
\text { significantly } \\
\text { improved survival in } \\
\text { comparison to BSC. }\end{array}$ \\
\hline $\begin{array}{l}\text { Casaretto } \\
\mathrm{L}[60]\end{array}$ & $\begin{array}{lr}\text { Braz J } & \text { Med } \\
\text { Biol } & \text { Res } \\
(2006) & \end{array}$ & $\begin{array}{l}\text { Chemotherapy } \\
\text { vs. BSC }\end{array}$ & $\begin{array}{l}\text { OS: 3-, 6-mo: } \\
\text { statistically similar. } \\
\text { 12-mo: favor } \\
\text { chemotherapy. }\end{array}$ & $\begin{array}{l}\text { Objective } \\
\text { RR: } \\
\text { statistically } \\
\text { similar. }\end{array}$ & NA. & $\begin{array}{l}\text { Chemotherapy } \\
\text { increased the } 1-y \text { SR, } \\
\text { provided a longer } \\
\text { symptom-free period } \\
\text { of } 6 \text { months and } \\
\text { an improvement in } \\
\text { quality of life. }\end{array}$ \\
\hline $\begin{array}{l}\text { Wagner } \\
\text { AD [59] }\end{array}$ & $\begin{array}{l}\text { J Clin Oncol } \\
(2006)\end{array}$ & $\begin{array}{l}\text { (Include } \\
\text { chemotherapy } \\
\text { vs. BSC). }\end{array}$ & Favor chemotherapy. & NA. & NA. & - \\
\hline
\end{tabular}

\section{D2 vs. D4 lymphadenectomy}

Two meta-analyses compared D2 lymphadenectomy versus $\mathrm{D} 2+$ para-aortic nodal dissection, which is also called D4 lymphadenectomy. Both of them demonstrated that there were no significant differences between two groups in 5-year OS or 5-year survival rate [53, 54]. One of them showed that the 5-year recurrence was also statistically similar between two groups [54] while the other did not report relevant data.

Both RCT and non-RCT studies were included in the meta-analysis by Wang and all studies included in Zhang's article were RCT studies.

The meta-analysis by Wang had a larger number of included studies than those by Zhang (11 versus 4) (Supplementary Table S9). Notably, there was an overlap of included studies between the two meta-analyses by Wang and Zhang. All studies that were included in the meta-analysis by Zhang were also covered by the metaanalysis by Wang.
Given its superiority in the quantity of RCT studies, the results of the meta-analysis by Wang might be reliable. In details, D2 plus PAND (D4) could be performed as safely as standard D2 resection without increasing postoperative mortality but failed to benefit OS in patients with AGC. However, D2 group was favored in terms of operation time and blood loss.

\section{D2 vs. D3 lymphadenectomy}

One meta-analysis compared the outcomes of D2 versus D3 lymphadenectomy. There was no significant difference between two groups regarding 5-year survival rate. So were postoperative mortality, anastomotic leakage, and pneumonia. Additionally, D2 dissection was favorable in terms of operation time, blood loss during surgery and postoperative pancreatic leakage. Owing to the limitation in statistically methods of the included studies, further rigorously controlled trials are required before conclusions on which procedure was more favorable could be made [48]. 
Table 7: Findings of meta-analyses: An overview of included studies regarding S-1-based therapy vs. 5-FU-based therapy

\begin{tabular}{|c|c|c|c|c|c|c|}
\hline $\begin{array}{l}\text { First } \\
\text { Author }\end{array}$ & $\begin{array}{l}\text { Journal } \\
\text { (Year) }\end{array}$ & Comparisons & OS & RR & $\begin{array}{l}\text { SR, DFS, RFS, } \\
\text { PFS, Recurrence, } \\
\text { TTF,TTP }\end{array}$ & Major comments \\
\hline $\begin{array}{ll}\text { Ter } & \text { Veer } \\
\text { E[7] } & \end{array}$ & $\begin{array}{l}\text { Gastric } \\
\text { Cancer } \\
(2016)\end{array}$ & $\begin{array}{l}\text { (Include S-1-based } \\
\text { therapy vs. 5-FU- } \\
\text { based therapy) }\end{array}$ & $\begin{array}{l}\text { Statistically } \\
\text { similar. }\end{array}$ & $\begin{array}{l}\text { Objective RR: } \\
\text { favor S-1- } \\
\text { based therapy. }\end{array}$ & $\begin{array}{l}\text { PFS: statistically } \\
\text { similar. }\end{array}$ & $\begin{array}{l}\text { S-1-based therapy } \\
\text { showed no difference } \\
\text { in survival, but the } \\
\text { toxicity profile of } \\
\text { S-1 was clearly more } \\
\text { advantageous in } \\
\text { Western patients. } \\
\end{array}$ \\
\hline $\begin{array}{l}\mathrm{Wu} \\
\mathrm{FL}[14]\end{array}$ & $\begin{array}{l}\text { Medicine } \\
\text { (Baltimore) } \\
(2015)\end{array}$ & $\begin{array}{l}\text { (Include S-1 based } \\
\text { therapy vs. 5-FU- } \\
\text { based therapy). }\end{array}$ & $\begin{array}{l}\text { Favor } \\
\text { S-1-based } \\
\text { therapy. }\end{array}$ & $\begin{array}{l}\text { Objective RR: } \\
\text { favor S-1- } \\
\text { based therapy. }\end{array}$ & $\begin{array}{l}\text { PFS: favor S-1-based } \\
\text { therapy. }\end{array}$ & $\begin{array}{l}\text { S-1-based } \\
\text { chemotherapy was } \\
\text { favorable to AGC } \\
\text { patients with better } \\
\text { clinical benefit } \\
\text { than 5-FU-based } \\
\text { chemotherapy. }\end{array}$ \\
\hline Yang J[24] & $\begin{array}{l}\text { World J } \\
\text { Gastroenterol } \\
(2014)\end{array}$ & $\begin{array}{l}\text { (Include S-1 based } \\
\text { therapy vs. 5-FU } \\
\text {-based therapy). }\end{array}$ & $\begin{array}{l}\text { Favor } \\
\text { S-1-based } \\
\text { regimens. }\end{array}$ & $\begin{array}{l}\text { Objective } \\
\text { RR: favor } \\
\text { S-1-based } \\
\text { regimens. }\end{array}$ & $\begin{array}{l}\text { PFS: statistically } \\
\text { similar. TTF: favor } \\
\text { S-1-based regimens }\end{array}$ & $\begin{array}{l}\text { S-1-based } \\
\text { chemotherapy } \\
\text { prolonged OS and } \\
\text { TTF, and induced } \\
\text { less leukopenia and } \\
\text { stomatitis. }\end{array}$ \\
\hline Li DH[19] & $\begin{array}{l}\text { Tumour Biol } \\
(2014)\end{array}$ & $\begin{array}{l}\text { S-1-based versus } \\
\text { 5-FU-based } \\
\text { chemotherapy }\end{array}$ & $\begin{array}{l}\text { Favor } \\
\text { S-1-based } \\
\text { therapy }\end{array}$ & $\begin{array}{l}\text { Overall RR: } \\
\text { statistically } \\
\text { similar. }\end{array}$ & $\begin{array}{l}\text { TTF: statistically } \\
\text { similar. }\end{array}$ & $\begin{array}{l}\text { S-1-based therapy } \\
\text { was favorable in OS } \\
\text { and safety profile as } \\
\text { first-line treatment in } \\
\text { AGC. It was prone } \\
\text { to improving overall } \\
\text { RR and TTF, though } \\
\text { the difference was not } \\
\text { significant. } \\
\end{array}$ \\
\hline Liu H[21] & $\begin{array}{l}\text { Medicine } \\
\text { (Baltimore) } \\
(2014)\end{array}$ & $\begin{array}{l}\text { S-1+ paclitaxel vs. } \\
\text { 5-FU+ paclitaxel }\end{array}$ & NA. & $\begin{array}{l}\text { Overall RR: } \\
\text { statistically } \\
\text { similar. }\end{array}$ & $\begin{array}{l}\text { Median PFS: favor } \\
\text { S-1+ paclitaxel } \\
\text { therapy. 6-mo } \\
\text { PFS, TTF, TTP: } \\
\text { statistically similar. }\end{array}$ & $\begin{array}{l}\text { S-1+ paclitaxel } \\
\text { therapy was a good } \\
\text { alternative strategy } \\
\text { for patients who } \\
\text { could not tolerate } \\
\text { a continuous } \\
\text { intravenous infusion. } \\
\end{array}$ \\
\hline $\begin{array}{l}\text { Huang } \\
\text { J[42] }\end{array}$ & $\begin{array}{l}\text { Med } \\
\text { (2011) }\end{array}$ & $\begin{array}{l}\text { S-1-based therapy } \\
\text { vs. 5-FU-based } \\
\text { therapy }\end{array}$ & $\begin{array}{l}\text { Favor } \\
\text { S-1-based } \\
\text { therapy. }\end{array}$ & $\begin{array}{l}\text { Overall RR: } \\
\text { statistically } \\
\text { similar. }\end{array}$ & NA. & $\begin{array}{l}\text { S-1-based therapy } \\
\text { significantly } \\
\text { improved OS. Overall } \\
\text { RR and safety profile } \\
\text { were considerable } \\
\text { between two groups. }\end{array}$ \\
\hline
\end{tabular}

\section{Palliative gastrectomy vs. non-palliative gastrectomy}

One meta-analysis compared the outcomes of palliative gastrectomy versus non-palliative gastrectomy. Palliative gastrectomy for patients with incurable AGC was associated with longer OS, especially for patients with stage M1 GC [36].

\section{Palliative gastrectomy with vs. without hepatic resection}

One meta-analysis compared the outcomes of palliative gastrectomy combined with hepatic resection versus gastrectomy without hepatic resection in AGC patients with liver metastasis. The meta-analysis confirmed that palliative gastrectomy combined with hepatectomy might provide better OS than palliative gastrectomy only [36].

\section{Drain vs. no-drain after gastrectomy}

One meta-analysis evaluated drain versus no-drain after gastrectomy. The study did not report survival data. There were no significant differences between the drain and no-drain groups in terms of wound infection, postoperative pulmonary infection, intra-abdominal abscess, mortality, number of postoperative days until passing of flatus and initiation of soft diet. Besides, the results favored no drain after gastrectomy regarding 
Table 8: Findings of meta-analyses: An overview of included studies regarding S-1-based vs. capecitabine-based therapy

\begin{tabular}{|c|c|c|c|c|c|c|c|}
\hline $\begin{array}{l}\text { First } \\
\text { Author }\end{array}$ & $\begin{array}{l}\text { Journal } \\
\text { (Year) }\end{array}$ & Comparisons & OS & $\mathbf{R R}$ & $\begin{array}{l}\text { SR, DFS, } \\
\text { RFS, PFS, } \\
\text { Recurrence, } \\
\text { TTF,TTP }\end{array}$ & Other & $\begin{array}{l}\text { Major } \\
\text { comments }\end{array}$ \\
\hline $\begin{array}{l}\text { Ter Veer } \\
E[7]\end{array}$ & $\begin{array}{l}\text { Gastric } \\
\text { Cancer } \\
\text { (2016) }\end{array}$ & $\begin{array}{l}\text { (Include S-1- } \\
\text { based vs. } \\
\text { capecitabine- } \\
\text { based therapy) }\end{array}$ & $\begin{array}{l}\text { Statistically } \\
\text { similar. }\end{array}$ & $\begin{array}{l}\text { Objective } \\
\text { RR: } \\
\text { statistically } \\
\text { similar. }\end{array}$ & $\begin{array}{l}\text { PFS: } \\
\text { statistically } \\
\text { similar. }\end{array}$ & $\begin{array}{l}\text { Grade 3-4 } \\
\text { neutropenia and } \\
\text { grade 1-2 hand- } \\
\text { foot syndrome: } \\
\text { lower in } \\
\text { S-1-based } \\
\text { therapy. Febrile } \\
\text { neutropenia, } \\
\text { serious AEs, } \\
\text { toxicity- } \\
\text { related deaths: } \\
\text { statistically } \\
\text { similar. } \\
\end{array}$ & $\begin{array}{l}\text { S-1-based } \\
\text { therapy showed } \\
\text { no difference } \\
\text { in survival but } \\
\text { showed a better } \\
\text { toxicity profile } \\
\text { compared with } \\
\text { capecitabine } \\
\text { based therapy. }\end{array}$ \\
\hline $\begin{array}{l}\mathrm{Wu} \\
\mathrm{FL}[14]\end{array}$ & $\begin{array}{l}\text { Medicine } \\
\text { (Baltimore) } \\
\text { (2015) }\end{array}$ & $\begin{array}{l}\text { (Include } \\
\text { S-1- based vs. } \\
\text { capecitabine- } \\
\text { based therapy) }\end{array}$ & $\begin{array}{l}\text { Statistically } \\
\text { similar. }\end{array}$ & $\begin{array}{l}\text { Overall } \\
\text { RR: } \\
\text { statistically } \\
\text { similar. }\end{array}$ & $\begin{array}{l}\text { PFS: } \\
\text { statistically } \\
\text { similar. }\end{array}$ & $\begin{array}{l}\text { Overall grade } \\
3-4 \text { toxicity: } \\
\text { statistically } \\
\text { similar. Grade } 3 \\
\text { to } 4 \text { toxicity of } \\
\text { diarrhea: lower } \\
\text { in S-1-based } \\
\text { therapy. }\end{array}$ & $\begin{array}{l}\text { S-1-based } \\
\text { chemotherapy } \\
\text { had equivalent } \\
\text { antitumor } \\
\text { compared with } \\
\text { capecitabine } \\
\text { based therapy. }\end{array}$ \\
\hline $\begin{array}{l}\text { Yang } \\
\mathrm{J}[24]\end{array}$ & $\begin{array}{l}\text { World J } \\
\text { Gastroenterol } \\
\text { (2014) }\end{array}$ & $\begin{array}{l}\text { (Include S-1 } \\
\text { based vs. } \\
\text { capecitabine- } \\
\text { based therapy). }\end{array}$ & $\begin{array}{l}\text { Statistically } \\
\text { similar. }\end{array}$ & $\begin{array}{l}\text { Objective } \\
\text { RR, TTF: } \\
\text { statistically } \\
\text { similar. }\end{array}$ & $\begin{array}{l}\text { PFS: } \\
\text { statistically } \\
\text { similar. }\end{array}$ & $\begin{array}{l}\text { Grade } 3 \\
\text { or } 4 \text { AEs: } \\
\text { statistically } \\
\text { similar. }\end{array}$ & $\begin{array}{l}\text { S-1 and } \\
\text { capecitabine } \\
\text { could be used } \\
\text { for AGC } \\
\text { interchangeably. }\end{array}$ \\
\hline $\begin{array}{l}\mathrm{He} \\
\mathrm{MM}[30]\end{array}$ & $\begin{array}{l}\text { PLoS One } \\
\text { (2013) }\end{array}$ & $\begin{array}{l}\text { S-1-based vs. } \\
\text { capecitabine- } \\
\text { based } \\
\text { chemotherapy } \\
\text { as first-line } \\
\text { treatment. }\end{array}$ & $\begin{array}{l}\text { OS, Survival } \\
\text { probability } \\
(0.5-, \\
1-, 2-\text { y): } \\
\text { statistically } \\
\text { similar. }\end{array}$ & $\begin{array}{l}\text { Overall } \\
\text { RR: } \\
\text { statistically } \\
\text { similar. }\end{array}$ & $\begin{array}{l}\text { TTP: } \\
\text { statistically } \\
\text { similar. } \\
\text { Progression- } \\
\text { free } \\
\text { probability: } \\
\text { 3-mo, 6-mo: } \\
\text { statistically } \\
\text { similar. }\end{array}$ & $\begin{array}{l}\text { Grade 3-4 } \\
\text { hematological } \\
\text { and non- } \\
\text { hematological } \\
\text { toxicities: } \\
\text { statistically } \\
\text { similar (except } \\
\text { hand-foot } \\
\text { syndrome: less } \\
\text { in S-1-based } \\
\text { chemotherapy). }\end{array}$ & $\begin{array}{l}\text { S-1-based } \\
\text { chemotherapy } \\
\text { was associated } \\
\text { with non- } \\
\text { inferior } \\
\text { antitumor } \\
\text { efficacy and } \\
\text { better safety } \\
\text { profile, We } \\
\text { recommended } \\
\text { S-1 and } \\
\text { capecitabine } \\
\text { could be used } \\
\text { interchangeably } \\
\text { for AGC, at } \\
\text { least in Asia. }\end{array}$ \\
\hline
\end{tabular}

postoperative complications and the length of hospital stay [44].

\section{Chemotherapy}

\section{Chemotherapy vs. basic supportive care (BSC)}

Six meta-analyses compared the outcomes of chemotherapy with BSC $[9,18,31,51,59,60]$. Five of them reported OS without detailed time points showing that OS was improved by chemotherapy $[9,18,31,51$, 59]. Among them, one meta-analysis comparing six regimens of chemotherapy with BSC demonstrated that OS was only favorable in paclitaxel and ramucirumab
+ paclitaxel vs. BSC while the other four chemotherapy regimens showed no statistical difference [9]. Another meta-analysis revealed that the 3- and 6- month OS was statistically similar between chemotherapy and BSC. However, chemotherapy was favorable in terms of 12-month OS [60]. One meta-analysis reported response data, which indicated that the objective response rate (RR) was statistically similar between the two groups [60]. Beside, one of them favored chemotherapy regarding time to progression (TTP) [51]. Chemotherapy brought benefit in terms of symptom-free period, quality of life and tumor mass reduction according to the results of one of the metaanalyses [60].

Only RCT studies, rather than non-RCTs were 
Table 9: Findings of meta-analyses: An overview of included studies regarding S-1-based and combination therapy

\begin{tabular}{|c|c|c|c|c|c|c|c|}
\hline $\begin{array}{l}\text { First } \\
\text { Author }\end{array}$ & $\begin{array}{l}\text { Journal } \\
\text { (Year) }\end{array}$ & Comparisons & OS & $\mathbf{R R}$ & \begin{tabular}{|l|} 
SR, DFS, \\
RFS, PFS, \\
Recurrence, \\
TTF,TTP \\
\end{tabular} & Other & $\begin{array}{l}\text { Major } \\
\text { comments }\end{array}$ \\
\hline \multicolumn{8}{|c|}{ S-1 based vs. cisplatin based therapy } \\
\hline $\begin{array}{l}\text { Wu } \\
\text { FL[14] }\end{array}$ & $\begin{array}{l}\text { Medicine } \\
\text { (Baltimore) } \\
(2015)\end{array}$ & $\begin{array}{ll}\text { Include } & \text { S-1 } \\
\text {-based } & \text { vs. } \\
\text { cisplatin-based } \\
\text { therapy) }\end{array}$ & $\begin{array}{l}\text { Statistically } \\
\text { similar. }\end{array}$ & $\begin{array}{l}\text { Objective } \\
\text { RR: } \\
\text { statistically } \\
\text { similar. } \\
\end{array}$ & $\begin{array}{l}\text { PFS: } \\
\text { statistically } \\
\text { similar. }\end{array}$ & NA. & - \\
\hline \multicolumn{8}{|c|}{ S-1 based combination therapy vs. S-1 monotherapy } \\
\hline $\begin{array}{l}\text { Ter Veer } \\
\text { E[7] }\end{array}$ & $\begin{array}{l}\text { Gastric } \\
\text { Cancer } \\
(2016)\end{array}$ & $\begin{array}{l}\text { (Include } \\
\text { S-1-based } \\
\text { combination } \\
\text { therapy vs. S-1 } \\
\text { monotherapy) }\end{array}$ & $\begin{array}{l}\text { Favor S-1 } \\
\text { combination } \\
\text { therapy. }\end{array}$ & $\begin{array}{l}\text { Objective } \\
\text { RR: } \\
\text { favor S-1 } \\
\text { combination } \\
\text { therapy. }\end{array}$ & $\begin{array}{l}\text { PFS: } \\
\text { favor S-1 } \\
\text { combination } \\
\text { therapy. }\end{array}$ & $\begin{array}{l}\text { AEs: higher } \\
\text { in S-1 } \\
\text { combination } \\
\text { therapy. }\end{array}$ & $\begin{array}{l}\text { S-1 } \\
\text { combination } \\
\text { therapy } \\
\text { was more } \\
\text { efficacious } \\
\text { than S-1 } \\
\text { monotherapy. }\end{array}$ \\
\hline $\begin{array}{l}\text { Liu } \\
\text { GF[20] }\end{array}$ & $\begin{array}{l}\text { World J } \\
\text { Gastroenterol } \\
(2014)\end{array}$ & $\begin{array}{l}\text { S-1-based } \\
\text { combination } \\
\text { therapy vs. S-1 } \\
\text { monotherapy }\end{array}$ & $\begin{array}{l}\text { Favor S-1 } \\
\text { combination } \\
\text { therapy. }\end{array}$ & $\begin{array}{l}\text { Overall RR: } \\
\text { favor S-1 } \\
\text { combination } \\
\text { therapy. }\end{array}$ & $\begin{array}{l}\text { PFS: } \\
\text { favor S-1 } \\
\text { combination } \\
\text { therapy. }\end{array}$ & $\begin{array}{l}\text { Grade 3-4 } \\
\text { leucopenia, } \\
\text { neutropenia, } \\
\text { diarrhea: } \\
\text { higher in S-1 } \\
\text { combination } \\
\text { therapy. }\end{array}$ & $\begin{array}{l}\text { S-1-based } \\
\text { combination } \\
\text { therapy was } \\
\text { superior to } \\
\text { monotherapy } \\
\text { in terms of } \\
\text { OS, PFS and } \\
\text { overall RR. }\end{array}$ \\
\hline $\begin{array}{l}\text { Wu } \\
\text { JR[23] }\end{array}$ & $\begin{array}{l}\text { Tumour Biol } \\
(2014)\end{array}$ & $\begin{array}{l}\text { S-1-based } \\
\text { combination } \\
\text { therapy vs. S-1 } \\
\text { monotherapy. }\end{array}$ & $\begin{array}{l}\text { Favor S-1 } \\
\text { combination } \\
\text { therapy. }\end{array}$ & $\begin{array}{l}\text { Objective } \\
\text { RR: } \\
\text { favor S-1 } \\
\text { combination } \\
\text { therapy. }\end{array}$ & $\begin{array}{l}\text { PFS: } \\
\text { favor S-1 } \\
\text { combination } \\
\text { therapy. }\end{array}$ & $\begin{array}{l}\text { Grade } 3 / 4 \\
\text { toxicity event: } \\
\text { higher in S-1 } \\
\text { combination } \\
\text { therapy. }\end{array}$ & $\begin{array}{l}\text { For the Asian } \\
\text { population, } \\
\text { S-1 } \\
\text { combination } \\
\text { therapy } \\
\text { improved } \\
\text { OS and PFS } \\
\text { and enhanced } \\
\text { objective } \\
\text { RR. The } \\
\text { safety profile } \\
\text { was poorer } \\
\text { in patients } \\
\text { with S-1 } \\
\text { combination } \\
\text { therapy }\end{array}$ \\
\hline
\end{tabular}

included in the six meta-analyses. The meta-analysis by Badiani had the largest number of included studies ( $n=$ $7)$, followed by the meta-analysis by Casaretto $(n=5)$ (Supplementary Table S10). Notably, there was an overlap of included studies between the two meta-analyses by Wagner AD published in 2010 and 2006, respectively. All related studies included in the meta-analysis by Wagner in 2010 were also covered by the one he wrote in 2006 .

Given its superiority in the quantity of RCT studies, the results of the study by Badiani might be more reliable. In details, both paclitaxel monotherapy and ramucirumab plus paclitaxel therapy determined a significant prolongation in survival as compared with BSC.

\section{S-1-based therapy vs. 5-FU-based therapy}

Six meta-analyses compared S-1-based therapy with 5-FU-based therapy [7, 14, 19, 21, 24, 42]. Five of them compared OS [7, 14, 19, 24, 42]. Among them, four of them favored S-1-based therapy [14, 19, 24, 42] while one meta-analysis showed that there was no statistically difference between two groups in terms of OS [7]. As for objective RR or overall RR, three of them favored S-1 in terms of objective RR [7, 14, 24] while the other three meta-analyses suggested that overall RR was statistically similar between the two groups [19, 21, 42]. Four metaanalyses reported PFS [7, 14, 21, 24,]. Two of them showed that the PFS was statistically similar between the S-1-based and 5-FU-based therapy [7, 24]; one favored S-1-based therapy over 5-FU-based therapy [14]; the other one revealed that $\mathrm{S}-1$-based therapy was favorable in terms of median PFS but the 6-month PFS was statistically similar between two groups [21]. Three of them compared time to failure (TTF) [19, 21, 24]; one favored S-1-based regimen [24] while the other two showed that there were no significant differences between two groups [19, 
Table 10: Findings of meta-analyses: An overview of included studies regarding oxaliplatin-based vs. cisplatin-based therapy

\begin{tabular}{|c|c|c|c|c|c|c|c|}
\hline First Author & $\begin{array}{l}\text { Journal } \\
\text { (Year) }\end{array}$ & Comparisons & OS & RR & $\begin{array}{l}\text { SR, DFS, } \\
\text { RFS, PFS, } \\
\text { Recurrence, } \\
\text { TTF,TTP }\end{array}$ & Other & $\begin{array}{l}\text { Major } \\
\text { comments }\end{array}$ \\
\hline $\begin{array}{l}\text { Montagnani } \\
\text { F[46] }\end{array}$ & $\begin{array}{l}\text { Gastric } \\
\text { Cancer } \\
\text { (2011) }\end{array}$ & $\begin{array}{l}\text { Oxaliplatin vs. } \\
\text { cisplatin. }\end{array}$ & NA. & NA. & $\begin{array}{l}\text { PFS: favor } \\
\text { oxaliplatin. }\end{array}$ & $\begin{array}{l}\text { Neutropenia, } \\
\text { thromboembolic: } \\
\text { lower in } \\
\text { oxaliplation. } \\
\text { Neurotoxicity: in } \\
\text { higher } \\
\text { oxaliplation. } \\
\end{array}$ & $\begin{array}{l}\text { A small but } \\
\text { significant } \\
\text { survival } \\
\text { benefit of } \\
\text { oxaliplatin was } \\
\text { associated with } \\
\text { less toxicity } \\
\text { and better } \\
\text { tolerability, in } \\
\text { especially in } \\
\text { older patients } \\
\text { and when used } \\
\text { in two-drug, } \\
\text { bi-weekly } \\
\text { regimens. }\end{array}$ \\
\hline $\begin{array}{l}\text { Wagner } \\
\mathrm{AD}[51]\end{array}$ & $\begin{array}{l}\text { Cochrane } \\
\text { Database } \\
\text { Syst Rev } \\
(2010)\end{array}$ & $\begin{array}{l}\text { (Include } \\
\text { oxaliplatin- } \\
\text { vs. cisplatin- } \\
\text { containing } \\
\text { regimens). }\end{array}$ & $\begin{array}{l}\text { Statistically } \\
\text { similar }\end{array}$ & $\begin{array}{l}\text { Objective RR: } \\
\text { favor oxaliplatin } \\
\text {-based therapy. }\end{array}$ & $\begin{array}{l}\text { PFS:statistically } \\
\text { similar. }\end{array}$ & $\begin{array}{l}\text { Treatment related } \\
\text { death, treatment } \\
\text { discontinuation } \\
\text { due to toxicity: } \\
\text { statistically } \\
\text { similar. }\end{array}$ & $\begin{array}{l}\text { These results } \\
\text { confirmed the } \\
\text { non-inferiority } \\
\text { of oxaliplatin, } \\
\text { as compared } \\
\text { to cisplatin, in } \\
\text { the treatment } \\
\text { of AGC. }\end{array}$ \\
\hline Gong JF[55] & $\begin{array}{l}\text { Zhonghua } \\
\text { Yi Xue Za } \\
\text { Zhi (2009) }\end{array}$ & $\begin{array}{l}\text { Oxaliplatin- } \\
\text { based } \\
\text { chemotherapy } \\
\text { vs. cisplatin } \\
\text {-based } \\
\text { chemotherapy }\end{array}$ & $\begin{array}{l}\text { OS: 1-y: favor } \\
\text { oxaliplatin- } \\
\text { based } \\
\text { chemotherapy }\end{array}$ & $\begin{array}{l}\text { Objective } \\
\text { RR: favor } \\
\text { oxaliplatin- } \\
\text { based } \\
\text { chemotherapy. }\end{array}$ & NA. & $\begin{array}{l}\text { Peripheral } \\
\text { neurotoxicity: in } \\
\text { higher } \\
\text { oxaliplatin-based } \\
\text { chemotherapy; } \\
\text { Anemia/nusea/ } \\
\text { vomiting: higher } \\
\text { in cisplatin-based } \\
\text { chemotherapy. }\end{array}$ & $\begin{array}{l}\text { Oxaliplatin- } \\
\text { based } \\
\text { chemotherapy } \\
\text { was well- } \\
\text { tolerated and } \\
\text { more effective } \\
\text { than cisplatin } \\
\text { in AGC. }\end{array}$ \\
\hline
\end{tabular}

21]. Only one meta-analysis reported TTP that it was statistically similar between two groups when it comes to TTP [21]. According to one of the included meta-analyses, S-1-based therapy was favorable regarding the incidence of progressive disease and disease control rate while the incidence of stable disease was significant similar between two groups [21]. One meta-analysis evaluated drug-related toxicity based on sub-analysis. In the Western subgroup, S-1-based therapy demonstrated significantly lower rates of febrile neutropenia, toxicity-related deaths, grade 3-4 stomatitis and mucositis and grade 1-2 diarrhea, stomatitis and alopecia compared with 5-FU-based therapy; besides, the rates of grade 1-2 neutropenia and hand-foot syndrome were greater with S-1 than with 5-FU. In the Asian subgroup, S-1-based therapy showed a significantly increased incidence of grade 3-4 fatigue and grade 1-2 abdominal pain but a lower incidence of grade 1-2 neutropenia, nausea and weight loss compared with 5-FU-based therapy [7]. The incidence of febrile neutropenia, serious adverse event (AEs)or toxicityrelated deaths was statistically similar between both arms [7]. One meta-analysis favored S-1 therapy regarding grade 3/4 nausea [14] but another one meta-analysis showed that there was no significant difference between two groups [42]. Three meta-analyses reported stomatitis that two of them favored S-1-based therapy $[19,24]$ while the other one revealed no statistically difference between two groups [42]. Two meta-analyses reported grade $3 / 4$ neutropenia. Both of them favored S-1-based therapy $[14,42]$. One meta-analysis compared overall grade $3 / 4$ toxicity that there was no significant difference between S-1-based and 5-FU-based therapy [14].

Only RCT studies, rather than non-RCTs, were included in the six meta-analyses.

The meta-analysis by Ter had the largest number of included studies, followed by the meta-analyses by $\mathrm{Wu}$ and Li (8 versus 6 versus 6) (Supplementary Table S11). Notably, there was an overlap of included studies between the three meta-analyses by $\mathrm{Wu}, \mathrm{Li}$ and Ter. The included studies were completely same between the two metaanalyses by $\mathrm{Wu}$ and $\mathrm{Li}$, which were also covered by the meta-analysis by Ter.

Given its superiority in the quantity of RCT studies, the results of the meta-analysis by Ter might be reliable. In details, S-1-based therapy showed no difference in survival compared with 5-FU-based therapy, but the toxicity profile of S-1 was clearly more advantageous in Western patients. In addition, the two groups were statistically similar in 
Table 11: Findings of meta-analyses: An overview of included studies regarding capecitabine-based therapy vs.5-FU -based therapy

\begin{tabular}{|c|c|c|c|c|c|c|c|}
\hline $\begin{array}{l}\text { First } \\
\text { Author }\end{array}$ & $\begin{array}{l}\text { Journal } \\
\text { (Year) }\end{array}$ & Comparisons & OS & RR & $\begin{array}{l}\text { SR, DFS, } \\
\text { RFS, PFS, } \\
\text { Recurrence, } \\
\text { TTF,TTP }\end{array}$ & Other & $\begin{array}{l}\text { Major } \\
\text { comments }\end{array}$ \\
\hline $\mathrm{Xu} \mathrm{HB}[15]$ & $\begin{array}{l}\text { Eur J Clin } \\
\text { Pharmacol } \\
(2015)\end{array}$ & $\begin{array}{l}\text { Capecitabine+ } \\
\text { oxaliplatin } \\
\text { (XELOX) } \\
\text { 5-fluorouracil/ vs. } \\
\text { leucovorin+ } \\
\text { oxaliplatin } \\
\text { (FOLFOXs) }\end{array}$ & NA. & $\begin{array}{ll}\text { Overall } & \text { RR: } \\
\text { statistically } & \\
\text { similar } & \end{array}$ & NA. & $\begin{array}{l}\text { Clinical benefit } \\
\text { rate: statistically } \\
\text { similar. Nausea, } \\
\text { stomatitis, } \\
\text { diarrhea and } \\
\text { alopecia: lower } \\
\text { in capecitabine } \\
\text { regimen. Hand- } \\
\text { foot syndrome: } \\
\text { higher in } \\
\text { capecitabine } \\
\text { based regimen. }\end{array}$ & $\begin{array}{l}\text { Owing to } \\
\text { limited data and } \\
\text { potential bias } \\
\text { of the included } \\
\text { studies, further } \\
\text { rigorously } \\
\text { controlled trials } \\
\text { are required. }\end{array}$ \\
\hline Мa Y[40] & $\begin{array}{l}\text { J Clin } \\
\text { Pharm } \\
\text { Ther } \\
(2012)\end{array}$ & $\begin{array}{l}\text { Capecitabine-based } \\
\text { vs.5-FU-based } \\
\text { therapy. }\end{array}$ & $\begin{array}{l}\text { Favor } \\
\text { capecitabine- } \\
\text { based } \\
\text { chemotherapy }\end{array}$ & $\begin{array}{l}\text { Overall RR: favor } \\
\text { capecitabine- } \\
\text { based } \\
\text { chemotherapy }\end{array}$ & NA. & $\begin{array}{l}\text { Grade } 3 \text { or } 4 \\
\text { leukopenia } \\
\text {, stomatitis } \\
\text { and nausea } \\
\text { and vomiting, } \\
\text { hand-foot } \\
\text { syndrome: lower } \\
\text { in capecitabine- } \\
\text { based regimens ; } \\
\text { Haematological } \\
\text { toxicity: } \\
\text { statistically } \\
\text { similar }\end{array}$ & $\begin{array}{l}\text { Capecitabine } \\
\text { based } \\
\text { chemotherapy } \\
\text { strategies } \\
\text { showed } \\
\text { prolonged OS } \\
\text { and enhanced } \\
\text { overall } \\
\text { RR.AGC. } \\
\text { Asian patients } \\
\text { also showed } \\
\text { less grade } 3 / 4 \\
\text { gastrointestinal } \\
\text { toxicity with } \\
\text { the capecitabine } \\
\text { based regimens } \\
\text { compared with } \\
\text { Caucascian } \\
\text { patients. }\end{array}$ \\
\hline $\begin{array}{l}\text { Wagner } \\
\text { AD[51] }\end{array}$ & $\begin{array}{l}\text { Cochrane } \\
\text { Database } \\
\text { Syst Rev } \\
(2010)\end{array}$ & $\begin{array}{l}\text { (Include regimens } \\
\text { containing oral } \\
5-\mathrm{FU} \text { prodrugs } \\
\text { vs. intravenous } \\
\text { fluoropyrimidines). }\end{array}$ & $\begin{array}{l}\text { Statistically } \\
\text { similar }\end{array}$ & $\begin{array}{l}\text { Objective } \\
\text { RR: favor } \\
\text { capecitabine- } \\
\text { containing } \\
\text { regimen }\end{array}$ & $\begin{array}{l}\text { PFS: } \\
\text { statistically } \\
\text { similar. }\end{array}$ & $\begin{array}{l}\text { Treatment related } \\
\text { death, treatment } \\
\text { discontinuation } \\
\text { due to toxicity: } \\
\text { statistically } \\
\text { similar. }\end{array}$ & $\begin{array}{l}\text { Gastric cancer } \\
\text { patients with } \\
\text { adequate renal } \\
\text { function and } \\
\text { compliance } \\
\text { should be } \\
\text { treated with } \\
\text { capecitabine } \\
\text { instead of 5-FU. }\end{array}$ \\
\hline
\end{tabular}

terms of febrile neutropenia, serious AEs, toxicity-related death (Asian).

\section{S-1-based therapy vs. capecitabine-based therapy}

Four meta-analyses compared the outcomes of S-1-based therapy vs. capecitabine-based therapy [7, $14,24,30]$. As for OS, all the included meta-analyses demonstrated that the survival was statistically similar between the two groups. Besides, one meta-analysis that reported detailed survival data showed that the 0.5, 1-, and 2-year survival probability was statistically similar between the two groups [30]. As for objective $R R$ and overall RR, three of them found that that the two chemotherapy had statistically similar efficacy in terms of objective RR [7, 14, 24]; another meta-analysis that compared overall RR showed that there was no significant difference between two groups [30]. There were three meta-analyses to compare PFS. All of them demonstrated that PFS was statistically similar between two groups [7, 14, 24]. Additionally, another meta-analysis evaluated TTP and progression-free probability showed that TTP, 3- and 6-month progression-free probability was statistically similar between two regimens [30]. All the meta-analyses found that most of the grade 3-4 toxicity or adverse events AEs were statistically similar between two groups. When it comes to grade 1-2 hand-foot syndrome, one of them indicated that S-1-based therapy was favorable over capecitabine-based therapy [7].

Both RCTs and non-RCTs were included in the meta-analyses by $\mathrm{Wu}$ and $\mathrm{He}$ while only RCTs were included in the meta-analyses by Ter and Yang.

The meta-analysis by He had the largest number of included studies $(n=10)$ followed by the meta-analyses by Wu $(n=8)$, Ter $(n=3)$ and Yang $(n=2)$ (Supplementary Table S12). All studies that were included in the metaanalysis by Yang $(n=2)$ were also covered in the other 
Table 12: Findings of meta-analyses: An overview of included studies regarding CPT-11-based therapy vs. non CPT11-based therapy

\begin{tabular}{|c|c|c|c|c|c|c|c|}
\hline $\begin{array}{l}\text { First } \\
\text { Author }\end{array}$ & Journal (Year) & Comparisons & OS & RR & $\begin{array}{l}\text { SR, DFS, RFS, } \\
\text { PFS, Recurrence, } \\
\text { TTF,TTP }\end{array}$ & Other & $\begin{array}{l}\text { Major } \\
\text { comments }\end{array}$ \\
\hline Qi WX[35] & $\begin{array}{l}\text { Int J } \\
(2013)\end{array}$ & $\begin{array}{l}\text { CPT-11- } \\
\text { containing } \\
\text { Vs. non-CPT- } \\
\text { 11-containing } \\
\text { regimen. }\end{array}$ & $\begin{array}{l}\text { Favor CPT- } \\
\text { 11-containing } \\
\text { regiments. }\end{array}$ & $\begin{array}{l}\text { Overall RR: } \\
\text { statistically } \\
\text { similar. }\end{array}$ & $\begin{array}{l}\text { 1-y SR, TTF: } \\
\text { statistically similar; } \\
\text { PFS: favor CPT- } \\
\text { 11-containing } \\
\text { regiments. }\end{array}$ & $\begin{array}{l}\text { Grade } 3 / 4 \\
\text { fatigue: higher } \\
\text { in CPT-11- } \\
\text { containing } \\
\text { regimen. }\end{array}$ & $\begin{array}{l}\text { The study } \\
\text { provided strong } \\
\text { evidence for a } \\
\text { survival benefit } \\
\text { of CPT-11- } \\
\text { containing } \\
\text { regimen as first- } \\
\text { line treatment for } \\
\text { AGC. }\end{array}$ \\
\hline $\begin{array}{l}\text { Wang } \\
\text { DL[52] }\end{array}$ & $\begin{array}{l}\text { World J } \\
\text { Gastroenterol } \\
(2010)\end{array}$ & $\begin{array}{l}\text { CPT-11-based } \\
\text { chemotherapy } \\
\text { vs. non CPT- } \\
\text { 11-based } \\
\text { chemotherapy. }\end{array}$ & $\begin{array}{l}\text { Statistically } \\
\text { similar }\end{array}$ & $\begin{array}{l}\text { Overall RR: } \\
\text { statistically } \\
\text { similar. }\end{array}$ & $\begin{array}{l}\text { TTF: favor CPT- } \\
\text { 11-containing } \\
\text { chemotherapy }\end{array}$ & $\begin{array}{l}\text { Grade } 3 / 4 \\
\text { haemotological } \\
\text { toxicity and } \\
\text { gastrointestinal } \\
\text { toxicity: lower } \\
\text { in CPT-11- } \\
\text { containing }\end{array}$ & $\begin{array}{l}\text { CPT-11-based } \\
\text { therapy was } \\
\text { advantageous } \\
\text { over non } \\
\text { CPT-11-based } \\
\text { chemotherapy } \\
\text { for TTF with } \\
\text { no significant } \\
\text { toxicity. }\end{array}$ \\
\hline $\begin{array}{l}\text { Wagner } \\
\text { AD[51] }\end{array}$ & $\begin{array}{l}\text { Cochrane } \\
\text { Database Syst } \\
\text { Rev (2010) }\end{array}$ & $\begin{array}{l}\text { (Include CPT- } \\
\text { 11-containing } \\
\text { vs. non-CPT- } \\
\text { 11-containing } \\
\text { regimen). }\end{array}$ & $\begin{array}{l}\text { Statistically } \\
\text { similar }\end{array}$ & $\begin{array}{l}\text { Objective } \\
\text { RR: } \\
\text { statistically } \\
\text { similar. }\end{array}$ & $\begin{array}{l}\text { PFS: statistically } \\
\text { similar. }\end{array}$ & $\begin{array}{l}\text { Treatment } \\
\text { related death, } \\
\text { treatment } \\
\text { discontinuation } \\
\text { due to toxicity: } \\
\text { statistically } \\
\text { similar. }\end{array}$ & $\begin{array}{l}\text { CPT-11- } \\
\text { containing } \\
\text { regimens should } \\
\text { be considered as } \\
\text { a true and at least } \\
\text { equally effective } \\
\text { alternative to } \\
\text { platinum-based } \\
\text { combinations in } \\
\text { first-line therapy }\end{array}$ \\
\hline $\begin{array}{l}\text { Wagner } \\
\text { AD[59] }\end{array}$ & $\begin{array}{l}\mathrm{J} \text { Clin Oncol } \\
(2006)\end{array}$ & $\begin{array}{l}\text { (Include CPT- } \\
11-\text { containing } \\
\text { vs. non CPT- } \\
\text { 11- containing } \\
\text { therapy). }\end{array}$ & $\begin{array}{l}\text { Statistically } \\
\text { similar }\end{array}$ & NA. & NA. & $\begin{array}{l}\text { Treatment } \\
\text { related death: } \\
\text { statistically } \\
\text { similar. }\end{array}$ & $\begin{array}{l}\text { CPT-11- } \\
\text { containing } \\
\text { regimens } \\
\text { exhibited a } \\
\text { benefit in } \\
\text { survival and a } \\
\text { lower rate of } \\
\text { treatment-related } \\
\text { deaths although } \\
\text { these differences } \\
\text { were statistically } \\
\text { non-significant. }\end{array}$ \\
\hline
\end{tabular}

three meta-analyses. Besides, all studies included in the meta-analysis by $\mathrm{Wu}$ were also covered by the article of He.

Given its superiority in the quantity of RCT studies, the results of the meta-analyses by $\mathrm{He}$ and $\mathrm{Wu}$ might be reliable. The results of them were consistent with each other. In details, S-1-based chemotherapy was associated with non-inferior antitumor efficacy and better safety profile, compared with capecitabine-based therapy. It was recommended that S-1 and capecitabine could be used interchangeably for AGC, at least in Asia. Besides, Grade 3 to 4 hematological toxicities between two groups were statistically similar except hand-foot syndrome, which was less in S-1-based therapy.

\section{S-1-based therapy $v$ s. cisplatin-based therapy}

The efficacy of S-1-based and cisplatin-based therapy were evaluated in one meta-analysis. OS, objective RR and PFS were statistically similar between two groups. The meta-analysis did not report other survival data [14].

\section{S-1-based combination therapy vs. S-1 monotherapy}

Three meta-analyses compared the outcomes of S-1based combination and momotherapy. All the included meta-analyses indicated that S-1 combination therapy was superior to monotherapy in terms of OS and PFS [7, $20,23]$. As for RR, two of them favored S-1 combination therapy in objective RR [7, 23] while another one metaanalysis indicated that $\mathrm{S}-1$ was favorable regarding overall RR [20]. All the meta-analyses demonstrated that S-1 combination therapy was associated with higher incidence 
Table 13: Findings of meta-analyses: An overview of included studies regarding platinum-based and vs. cisplatin -based therapy

\begin{tabular}{|c|c|c|c|c|c|c|c|}
\hline $\begin{array}{l}\text { First } \\
\text { Author }\end{array}$ & $\begin{array}{l}\text { Journal } \\
\text { (Year) }\end{array}$ & Comparisons & OS & $\mathbf{R R}$ & $\begin{array}{l}\text { SR, DFS, } \\
\text { RFS, PFS, } \\
\text { Recurrence, } \\
\text { TTF,TTP }\end{array}$ & Other & Major comments \\
\hline \multicolumn{8}{|c|}{ Platinum based vs. non-platinum based therapy } \\
\hline $\begin{array}{l}\text { Chen } \\
\text { WW[28] }\end{array}$ & $\begin{array}{l}\text { PLoS One } \\
(2013)\end{array}$ & $\begin{array}{l}\text { Platinum-based } \\
\text { vs. non platinum } \\
\text {-based therapy }\end{array}$ & $\begin{array}{l}\text { Platinum- } \\
\text { based } \\
\text { vs. old- } \\
\text { generation } \\
\text { agents: favor } \\
\text { platinum- } \\
\text { based } \\
\text { therapy; } \\
\text { Platinum- } \\
\text { based vs. } \\
\text { g new- } \\
\text { generation } \\
\text { agents: } \\
\text { statistically } \\
\text { similar. }\end{array}$ & $\begin{array}{l}\text { RR: platinum- } \\
\text { based vs. old- } \\
\text { generation } \\
\text { agents: favor } \\
\text { platinum- } \\
\text { based therapy; } \\
\text { Platinum- } \\
\text { based vs. } \\
\text { g gew- } \\
\text { generation } \\
\text { agents: } \\
\text { statistically } \\
\text { similar. }\end{array}$ & NA. & $\begin{array}{l}\text { Hematological } \\
\text { toxicity, non- } \\
\text { hematological higher } \\
\text { toxicity: platinum- } \\
\text { in regimens. } \\
\text { based platinum- } \\
\text { Thrombocytopenia } \\
\text { (only in plam } \\
\text { based therapy vs. old- } \\
\text { generation agents), } \\
\text { nephrotoxicity (only } \\
\text { in platinum-based } \\
\text { therapy vs. new- } \\
\text { generation agents), } \\
\text { toxic death rate: } \\
\text { statistically similar. }\end{array}$ & $\begin{array}{l}\text { New-generation } \\
\text { agent (S-1, taxanes } \\
\text { and irinotecan) } \\
\text { based combination } \\
\text { regimens achieved } \\
\text { similar RR and } \\
\text { OS as platinum- } \\
\text { based therapy } \\
\text { that had generally } \\
\text { higher side effects. } \\
\text { S-1, taxanes and } \\
\text { irinotecan seemed } \\
\text { to be valid options } \\
\text { for patients } \\
\text { with inoperable, } \\
\text { advanced gastric } \\
\text { cancer as first-line } \\
\text { chemotherapy. }\end{array}$ \\
\hline \multicolumn{8}{|c|}{ Cisplatin vs. non cisplatin chemotherapy } \\
\hline $\begin{array}{l}\text { Petrelli } \\
\text { F[33] }\end{array}$ & $\begin{array}{l}\text { PLoS One } \\
(2013)\end{array}$ & $\begin{array}{l}\text { Chemotherapy } \\
\text { with vs. without } \\
\text { cisplatin }\end{array}$ & $\begin{array}{l}\text { Favor } \\
\text { therapy } \\
\text { without } \\
\text { cisplatin. }\end{array}$ & $\begin{array}{l}\text { RR: favor } \\
\text { therapy } \\
\text { without } \\
\text { cisplatin }\end{array}$ & $\begin{array}{l}\text { PFS: favor } \\
\text { therapy } \\
\text { without } \\
\text { cisplatin }\end{array}$ & NA. & $\begin{array}{l}\text { New active } \\
\text { cytotoxic agents } \\
\text { instead of cisplatin } \\
\text { significantly } \\
\text { enhanced OS, } \\
\text { PFS, and RR in } \\
\text { first-line treatment } \\
\text { of metastatic GC. }\end{array}$ \\
\hline
\end{tabular}

of AEs or grade 3-4 toxicity event [7, 20,23].

Only RCTs studies, rather than non-RCTs were included in the meta-analyses by Ter and Liu. Both RCTs and non-RCTs were included in Wu's meta-analysis.

The meta-analysis by Ter had the largest number of included studies $(n=8)$ followed by the meta-analyses by $\mathrm{Wu}(n=6)$ and Liu $(n=4)$ (Supplementary Table S13). Notably, there was an overlap of included studies between the three meta-analyses. All studies that were included in the meta-analysis by Liu were covered in Ter's and Wu's meta-analyses.

Given its superiority in the quantity of RCT studies, the results of the meta-analysis by Ter might be reliable. In details, S-1 combination therapy was more efficacious than S-1 monotherapy. However, AEs was higher and safety profile was poorer in S-1 combination therapy. This result was also confirmed by the other two meta-analyses.

\section{Oxaliplatin-based therapy vs. cisplatin-based therapy}

There were three meta-analyses to compare the outcomes of oxalipatin-based therapy versus cisplatin -based therapy [46, 51, 55]. Two of them evaluated OS. One found that survival was statistically similar between two groups [51]. But the other one favored oxalipatinbased therapy in terms of 1-year OS [55]. Two of them indicated that oxaliplatin-based therapy was superior to cisplatin-based therapy in terms of objective RR [51, 55] while one meta-analysis did not report RR data [46]. PFS was evaluated in two meta-analyses. One favored oxaliplatin while the other one showed no significant difference between two regimens. Oxaliplatin-based therapy was found to have higher neurotoxicity [46, 55] while cisplatin-based therapy had higher risk of neutropenia and thromboembolic events as well as anemia, nausea and vomiting events [55]. One meta-analysis revealed that the treatment related death and treatment discontinuation due to toxicity was statistically similar between two groups [51].

Only RCTs were included in two of the included meta-analyses except the one by Gong, which was not mentioned in the article.

The meta-analysis by Gong had the largest number of included studies $(n=16)$ (Supplementary Table S14). By comparison, the number of included studies was less than 10 in 2 other meta-analyses. Notably, there was an overlap of included studies between the meta-analyses by Wagner and Montagnani. All studies that were included in the meta-analysis by Wagner were also covered in the meta-analysis by Montagnani.

Given its superiority in the quantity of RCT studies, 
Table 14: Findings of meta-analyses: An overview of included studies regarding targeted chemotherapy

\begin{tabular}{|c|c|c|c|c|c|c|c|}
\hline First Author & $\begin{array}{l}\text { Journal } \\
\text { (Year) }\end{array}$ & Comparisons & OS & RR & $\begin{array}{l}\text { SR, DFS, } \\
\text { RFS, PFS, } \\
\text { Recurrence, } \\
\text { TTF,TTP }\end{array}$ & Other & $\begin{array}{l}\text { Major } \\
\text { comments }\end{array}$ \\
\hline $\begin{array}{l}\text { Ciliberto } \\
\mathrm{D}[10]\end{array}$ & $\begin{array}{l}\text { Cancer } \\
\text { Biol Ther } \\
(2015)\end{array}$ & $\begin{array}{l}\text { Targeted } \\
\text { therapy vs. } \\
\text { conventional } \\
\text { therapy }\end{array}$ & $\begin{array}{l}\text { Favor targeted } \\
\text { therapy of anti- } \\
\text { angiogenic and } \\
\text { HER2 but not } \\
\text { EGFR pathway }\end{array}$ & $\begin{array}{l}\text { RR: favor anti- } \\
\text { HER2 agents } \\
\text { but not for } \\
\text { anti-EGFR and } \\
\text { anti-angiogenic } \\
\text { agents. }\end{array}$ & $\begin{array}{l}\text { PFS: favor } \\
\text { targeted } \\
\text { therapy of anti- } \\
\text { angiogenic and } \\
\text { HER2 but not } \\
\text { EGFR pathway. }\end{array}$ & $\begin{array}{l}\text { Diarrhea: higher in } \\
\text { anti-HER2 agents. } \\
\text { Rash: higher in } \\
\text { anti-EGFR drugs. }\end{array}$ & $\begin{array}{l}\text { Targeted } \\
\text { therapy showed } \\
\text { a significant } \\
\text { survival benefit, } \\
\text { which can be } \\
\text { ascribed to } \\
\text { anti-angiogenic } \\
\text { and anti-HER2 } \\
\text { agents. }\end{array}$ \\
\hline $\begin{array}{l}\text { Iacovelli } \\
\mathrm{R}[18]\end{array}$ & $\begin{array}{l}\text { PLoS One } \\
(2014)\end{array}$ & \begin{tabular}{|l|} 
Targeted \\
therapy \\
vs. BSC or \\
traditional \\
chemotherapy
\end{tabular} & $\begin{array}{l}\text { OS: patients } \\
\text { ECOG=0: } \\
\text { statistically } \\
\text { similar; targeted } \\
\text { therapy vs. } \\
\text { chemotherapy: } \\
\text { favor chemotherapy. } \\
\text { chem patients } \\
\text { In ECOG } \geq 1 \text { : } \\
\text { with } \text { targeted therapy } \\
\text { vs. BSC: } \\
\text { favor targeted } \\
\text { therapy; targeted } \\
\text { therapy vs. } \\
\text { chemotherapy: } \\
\text { statistically } \\
\text { similar; }\end{array}$ & NA. & NA. & NA. & $\begin{array}{l}\text { In patients with } \\
\text { ECOG-PS = 0, } \\
\text { ramucirumab } \\
\text { and everolimus } \\
\text { did not report } \\
\text { a significant } \\
\text { survival benefit. } \\
\text { Any active } \\
\text { therapy over } \\
\text { BSC was more } \\
\text { effective on } \\
\text { patients with } \\
\text { ECOG-PS } \\
=1 \text { or more. } \\
\text { Patients with } \\
\text { symptomatic } \\
\text { disease not be } \\
\text { should not bediately } \\
\text { immed } \\
\text { excluded by } \\
\text { further lines of } \\
\text { therapy. }\end{array}$ \\
\hline Qi WX[22] & $\begin{array}{l}\text { Tumour } \\
\text { Biol } \\
(2014)\end{array}$ & $\begin{array}{l}\text { Anti-VEGF } \\
\text { agents vs. non } \\
\text { anti-VEGF } \\
\text { agents }\end{array}$ & $\begin{array}{l}\text { Favor anti-VEGF } \\
\text { therapy }\end{array}$ & $\begin{array}{l}\text { Objective RR: } \\
\text { favor anti-VEGF } \\
\text { therapy }\end{array}$ & $\begin{array}{l}\text { RFS: favor anti- } \\
\text { VEGF therapy }\end{array}$ & $\begin{array}{l}\text { Grade } 3 \text { or } 4 \\
\text { thrombocytopenia, } \\
\text { diarrhea, and } \\
\text { hypertension: } \\
\text { higher in anti- } \\
\text { VEGF therapy }\end{array}$ & $\begin{array}{l}\text { The anti-VEGF } \\
\text { therapy offered } \\
\text { a significant } \\
\text { survival benefit } \\
\text { in patients } \\
\text { with AGC, } \\
\text { especially for } \\
\text { those previously } \\
\text { treated patients. }\end{array}$ \\
\hline
\end{tabular}

the results of the meta-analysis by Montagnani might be more reliable. In details, oxaliplatin based therapy was associated with a small but significant survival benefit with less toxicity and better tolerability, especially in older patients and when used in two-drug, biweekly regimens.

\section{Capecitabine-based Capecitabine based therapy vs. 5-FU-based therapy}

Three meta-analyses compared the outcomes of capecitabine-based therapy versus 5 -FU-based therapy $[15,40,51]$. Capecitabine-based therapy was superior to 5-FU-based therapy in terms of OS according to one of the meta-analyses [40] while another meta-analysis showed that the survival was statistically between two groups [51]. And the other one did not report the survival data [15]. Besides, two of them found that capecitabine based therapy was associated with higher overall RR compared to 5 -FU based therapy $[40,51]$. On the contrary, the other one showed no significant difference between two groups [15]. PFS was evaluated in only one meta-analysis, which indicated that PFS was statistically similar between two groups. Two of meta-analyses showed that incidence of nausea and stomatitis was lower in capecitabine based therapy $[15,40]$. Higher frequency of hand-foot syndrome was observed in capecitabine-based therapy according to one of the meta-analyses [15] while the other one showed the opposite results [40]. And the other meta-analysis suggested that treatment related death as well as treatment discontinuation due to toxicity was statistically similar between two regimens [51].

Only RCTs, rather than non-RCTs were included in the three meta-analysis.

The meta-analyses by $\mathrm{Xu}$ had the largest number of included studies $(n=26)$ followed by the meta-analyses by Ma $(n=18)$ and by Wagner AD $(n=1)$ (Supplementary Table S15). Notably, there was an overlap of included 
Table 15: Findings of meta-analyses: An overview of included studies regarding combination (doublet/triplet) therapy vs. single/doublet therapy

\begin{tabular}{|c|c|c|c|c|c|c|c|}
\hline $\begin{array}{l}\text { First } \\
\text { Author }\end{array}$ & $\begin{array}{l}\text { Journal } \\
\text { (Year) }\end{array}$ & Comparisons & OS & RR & $\begin{array}{l}\text { SR, DFS, } \\
\text { RFS, PFS, } \\
\text { Recurrence, } \\
\text { TTF,TTP }\end{array}$ & Other & $\begin{array}{l}\text { Major } \\
\text { comments }\end{array}$ \\
\hline Zhang Y[8] & $\begin{array}{l}\text { Medicine } \\
\text { (Baltimore) } \\
\text { (2016) }\end{array}$ & $\begin{array}{l}\text { Doublet } \\
\text { combination } \\
\text { therapy vs. } \\
\text { single theapy }\end{array}$ & $\begin{array}{l}\text { Favor doublet } \\
\text { combination } \\
\text { therapy } \\
\text { targeted } \\
\text { agent+cytotoxic } \\
\text { chemotherapy } \\
\text { improved } \\
\text { OS, but not } \\
\text { for doublet } \\
\text { cytotoxic } \\
\text { chemotherapy) }\end{array}$ & $\begin{array}{l}\text { Objective RR: } \\
\text { favor doublet } \\
\text { combination } \\
\text { therapy }\end{array}$ & $\begin{array}{l}\text { PFS: favor } \\
\text { doublet } \\
\text { combination } \\
\text { therapy (also } \\
\text { significant in } \\
\text { targeted agent+ } \\
\text { chemotherapy } \\
\text { compared wth } \\
\text { single cytotoxic } \\
\text { agent). }\end{array}$ & $\begin{array}{l}\text { Grade } 3 / 4 \\
\text { myelosuppression, } \\
\text { toxicities, diarrhea, } \\
\text { fatigue: higher in } \\
\text { doublet combination } \\
\text { therapy; Grade 3/4 } \\
\text { thrombocytopenia, } \\
\text { nausea: statistically } \\
\text { similar. }\end{array}$ & $\begin{array}{l}\text { The addition } \\
\text { of targeted } \\
\text { agent to mono- } \\
\text { chemotherapy } \\
\text { as salvage } \\
\text { treatment for } \\
\text { pretreated AGC } \\
\text { patients provided } \\
\text { substantial } \\
\text { survival } \\
\text { benefits, while } \\
\text { no significant } \\
\text { survival benefits } \\
\text { were observed } \\
\text { in doublet } \\
\text { cytotoxic } \\
\text { chemotherapy } \\
\text { regimens. }\end{array}$ \\
\hline Liu N[39] & $\begin{array}{l}\text { Chin J Hos } \\
\text { Pharm } \\
\text { (2012) }\end{array}$ & $\begin{array}{l}\text { Triplet } \\
\text { chemotherapy } \\
\text { vs. doublet } \\
\text { chemotherapy }\end{array}$ & NA. & $\begin{array}{l}\text { Overall RR: } \\
\text { favor triplet } \\
\text { combination } \\
\text { chemotherapy. }\end{array}$ & NA. & $\begin{array}{l}\text { Effectiveness: favor } \\
\text { triplet combination } \\
\text { chemotherapy; } \\
\text { Grade 3/4 AEs: } \\
\text { statistically similar; }\end{array}$ & $\begin{array}{l}\text { Triplet } \\
\text { chemotherapy } \\
\text { was more } \\
\text { effective for } \\
\text { AGC patients } \\
\text { compared } \\
\text { to double } \\
\text { chemotherapy, } \\
\text { esp taxoid } \\
\text { based triplet } \\
\text { chemotherapy. }\end{array}$ \\
\hline $\begin{array}{l}\text { Wagner } \\
\mathrm{AD}[51]\end{array}$ & $\begin{array}{l}\text { Cochrane } \\
\text { Database } \\
\text { Syst Rev } \\
(2010)\end{array}$ & $\begin{array}{l}\text { (Include } \\
\text { combination } \\
\text { vs. } \\
\text { monotherapy) }\end{array}$ & $\begin{array}{l}\text { Favor } \\
\text { combination } \\
\text { chemotherapy. }\end{array}$ & $\begin{array}{l}\text { Objective } \\
\text { RR: favor } \\
\text { combination } \\
\text { chemotherapy. }\end{array}$ & $\begin{array}{l}\text { TTP: favor } \\
\text { combination } \\
\text { chemotherapy. }\end{array}$ & $\begin{array}{l}\text { Toxicity: higher } \\
\text { in combination } \\
\text { chemotherapy. }\end{array}$ & $\begin{array}{l}\text { Combination } \\
\text { chemotherapy } \\
\text { improved } \\
\text { survival } \\
\text { compared to } \\
\text { single-agent } \\
\text { therapy. }\end{array}$ \\
\hline $\begin{array}{l}\text { Wagner } \\
\mathrm{AD}[59]\end{array}$ & $\begin{array}{l}\text { J Clin } \\
\text { Oncol } \\
(2006)\end{array}$ & $\begin{array}{l}\text { (Include } \\
\text { combination } \\
\text { vs. } \\
\text { monotherapy) }\end{array}$ & $\begin{array}{l}\text { Favor } \\
\text { combination } \\
\text { chemotherapy. }\end{array}$ & NA. & NA. & $\begin{array}{l}\text { Toxicity: higher } \\
\text { in combination } \\
\text { therapy. Treatment } \\
\text { related death: } \\
\text { statistically similar. }\end{array}$ & $\begin{array}{l}\text { Combination } \\
\text { chemotherapy } \\
\text { improved } \\
\text { survival } \\
\text { compared to } \\
\text { single-agent } \\
\text { therapy. }\end{array}$ \\
\hline
\end{tabular}

studies between them.

Given its superiority in the quantity of RCTs studies, the results of the meta-analysis by $\mathrm{Xu}$ might be more reliable. In details, the evidence showed that XELOX (capecitabine-based therapy) might share similar efficacy as FOLFOXs (5-FU-based therapy) and reduced toxicities of chemotherapy in AGC therapy. However, owing to limited data and potential bias of the included studies, confirmation of these conclusions in rigorously controlled, randomized trials is required before more firm conclusions about this therapy can be drawn.

\section{Irinotecan (CPT-11)-based therapy vs. non CPT-11 -based}

The efficacy of CPT-11-based therapy versus nonCPT-11-based therapy was summarized in four metaanalyses $[35,51,52,59]$. When it comes to OS, three of them demonstrated that the survival was statistically similar [51, 52, 59] while one meta-analysis favored CPT-11-containing therapy in terms of OS but the 1-year survival rate did not show significant difference between two groups [35]. Additionally, statistically similar overall RR was observed in two meta-analyses [51, 52]. Another one found similar objective RR between two groups [35]. One meta-analysis did not report relevant data. Two meta-analyses reported PFS [35, 51]. One favored CPT11-containing regimen [35] while the other showed no 
Table 16: Findings of meta-analyses: An overview of included studies regarding FU/anthracycline/cisplatin combination therapy

\begin{tabular}{|c|c|c|c|c|c|c|c|}
\hline $\begin{array}{l}\text { First } \\
\text { Author }\end{array}$ & $\begin{array}{l}\text { Journal } \\
\text { (Year) }\end{array}$ & Comparisons & OS & RR & $\begin{array}{l}\text { SR, DFS, } \\
\text { RFS, PFS, } \\
\text { Recurrence, } \\
\text { TTF,TTP }\end{array}$ & Other & Major comments \\
\hline \multicolumn{8}{|c|}{ FU/anthracycline-containing combinations with vs. without cisplatin } \\
\hline $\begin{array}{l}\text { Wagner } \\
\mathrm{AD}[51]\end{array}$ & $\begin{array}{l}\text { Cochrane } \\
\text { Database } \\
\text { Syst Rev } \\
(2010)\end{array}$ & $\begin{array}{l}\text { (Include 5-FU/ } \\
\text { anthracycline-containing } \\
\text { combination therapy with } \\
\text { vs. without cisplatin). }\end{array}$ & $\begin{array}{l}\text { Favor } \\
\text { three-drug } \\
\text { combination. }\end{array}$ & NA. & NA. & NA. & $\begin{array}{l}\text { The comparisons } \\
\text { confirmed a statistically } \\
\text { significant advantage in } \\
\text { overall survival for the } \\
\text { three-drug combination } \\
\text { though this benefit was } \\
\text { achieved at the price of } \\
\text { significant toxicity }\end{array}$ \\
\hline $\begin{array}{l}\text { Wagner } \\
\text { AD[59] }\end{array}$ & $\begin{array}{l}\mathrm{J} \text { Clin } \\
\text { Oncol } \\
(2006)\end{array}$ & $\begin{array}{l}\text { (Include FU/ } \\
\text { anthracycline-containing } \\
\text { combination therapy with } \\
\text { vs. without cisplatin). }\end{array}$ & $\begin{array}{l}\text { Favor } \\
\text { three-drug } \\
\text { combination. }\end{array}$ & NA. & NA. & NA. & $\begin{array}{l}\text { Best survival results } \\
\text { were achieved with } \\
\text { three-drug regimens } \\
\text { containing FU, an } \\
\text { anthracycline, and } \\
\text { cisplatin. }\end{array}$ \\
\hline \multicolumn{8}{|c|}{ FU/cisplatin-containing regimens with vs. without anthracyclines } \\
\hline $\begin{array}{l}\text { Wagner } \\
\mathrm{AD}[51]\end{array}$ & $\begin{array}{l}\text { Cochrane } \\
\text { Database } \\
\text { Syst Rev } \\
(2010)\end{array}$ & $\begin{array}{l}\text { (Include 5-FU/cisplatin- } \\
\text { containing combination } \\
\text { therapy regimens with vs. } \\
\text { without anthracyclines). }\end{array}$ & $\begin{array}{l}\text { Favor } \\
\text { three-drug } \\
\text { combination. }\end{array}$ & NA. & NA. & NA. & $\begin{array}{l}\text { The comparisons } \\
\text { confirmed a statistically } \\
\text { significant advantage in } \\
\text { overall survival for the } \\
\text { three-drug combination } \\
\text { though this benefit was } \\
\text { achieved at the price of } \\
\text { significant toxicity }\end{array}$ \\
\hline $\begin{array}{l}\text { Wagner } \\
\text { AD[59] }\end{array}$ & $\begin{array}{l}\mathrm{J} \quad \text { Clin } \\
\text { Oncol } \\
(2006)\end{array}$ & $\begin{array}{ll}\text { (Include FU/cisplatin- } \\
\text { containing regimens } \\
\text { with vs. } & \text { without } \\
\text { anthracyclines)r } & \end{array}$ & $\begin{array}{l}\text { Favor } \\
\text { three-drug } \\
\text { combination. }\end{array}$ & NA. & NA. & NA. & $\begin{array}{l}\text { Best survival results } \\
\text { were achieved with } \\
\text { three-drug regimens } \\
\text { containing FU, an } \\
\text { anthracycline, and } \\
\text { cisplatin. }\end{array}$ \\
\hline
\end{tabular}

significant difference between two groups [51]. Besides, one meta-analysis reported TTF, which indicated that CPT-11-based therapy was favorable regarding TTF [52]. Treatment related death was reported in two metaanalyses. Both of them showed no significant difference between two regimens [51, 59]. Grade 3/4 fatigue was higher in CPT-11-based therapy according to the only related meta-analysis [35]. What's more, another metaanalysis revealed lower grade $3 / 4$ hematological toxicity and gastrointestinal toxicity in CPT-11-containing therapy [52].

Only RCT studies, rather than non-RCTs were included in the four meta-analyses.

The meta-analysis by Qi had the largest number of included studies $(n=10)$ (Supplementary Table S16). Notably, there was an overlap of included studies among the four articles.

Given its superiority in the quantity of RC studies, the results of the meta-analysis by Qi might be more reliable. In details, it provided strong evidence for a survival benefit of CPT-11-containing regimen as first-line treatment for AGC patients. The survival benefit of CPT11-based therapy for AGC was also confirmed in the other meta-analyses.

\section{Platinum-based therapy vs. non platinum-based therapy}

Platinum-based chemotherapy was evaluated in one meta-analysis. Non-platinum group were divided into two subgroups depending on whether new-generation agents such as $\mathrm{S}-1$, taxol or CPT-11 were contained. Platinumbased therapy achieved better outcomes in terms of OS and RR compared with old-generation therapies. On the contrary, when comparing to non-platinum regimens containing new-generation agents, platinum-based therapy did not seem to achieve better survival and enhanced response. Besides, Platinum-based therapy was associated with higher risk of most hematological and non-hematological toxicity events. In addition, toxic death rate was statistically similar between Platinum-based and 
Table 17: Findings of meta-analyses: An overview of included studies regarding docetaxel, lentinan and postoperative intravenous chemotherapy

\begin{tabular}{|c|c|c|c|c|c|c|c|}
\hline $\begin{array}{l}\text { First } \\
\text { Author }\end{array}$ & $\begin{array}{l}\text { Journal } \\
\text { (Year) }\end{array}$ & Comparisons & OS & $\mathbf{R R}$ & $\begin{array}{l}\text { SR, DFS, } \\
\text { RFS, PFS, } \\
\text { Recurrence, } \\
\text { TTF,TTP }\end{array}$ & Other & Major comments \\
\hline \multicolumn{8}{|c|}{ Docetaxel versus non docetaxel-containing regimens } \\
\hline $\begin{array}{l}\text { Wagner } \\
\mathrm{AD}[51]\end{array}$ & $\begin{array}{l}\text { Cochrane } \\
\text { Database } \\
\text { Syst Rev } \\
(2010)\end{array}$ & $\begin{array}{l}\text { (Include } \\
\text { docetaxel- } \\
\text { containing vs. } \\
\text { non docetaxel- } \\
\text { containing } \\
\text { regimens). }\end{array}$ & $\begin{array}{l}\text { Statistically } \\
\text { similar }\end{array}$ & $\begin{array}{l}\text { Objective } \\
\text { RR: } \\
\text { statistically } \\
\text { similar }\end{array}$ & $\begin{array}{l}\text { TTP: } \\
\text { statistically } \\
\text { similar. }\end{array}$ & $\begin{array}{l}\text { Treatment related } \\
\text { death, treatment } \\
\text { discontinuation } \\
\text { due to toxicity: } \\
\text { statistically similar. }\end{array}$ & $\begin{array}{l}\text { The clinical value } \\
\text { of docetaxel- } \\
\text { containing regimen } \\
\text { was regarded as } \\
\text { controversial. }\end{array}$ \\
\hline \multicolumn{8}{|c|}{ Chemotherapy regimens with vs. without lentinan administration } \\
\hline Oba K[56] & $\begin{array}{l}\text { Anticancer } \\
\text { Res (2009) }\end{array}$ & $\begin{array}{l}\text { Chemotherapy } \\
\text { regimens with } \\
\text { vs. without } \\
\text { lentinan } \\
\text { administration }\end{array}$ & $\begin{array}{l}\text { Favor with } \\
\text { lentinan } \\
\text { combination } \\
\text { therapy. }\end{array}$ & NA. & NA. & NA. & $\begin{array}{l}\text { The addition of } \\
\text { lentinan to standard } \\
\text { chemotherapy } \\
\text { offered a significant } \\
\text { advantage over } \\
\text { chemotherapy alone } \\
\text { in terms of survival. }\end{array}$ \\
\hline \multicolumn{8}{|c|}{ EPIPC vs. early postoperative intravenous chemotherapy } \\
\hline $\begin{array}{l}\text { Zhang } \\
\text { YL[54] }\end{array}$ & $\begin{array}{l}\text { Chin Gen } \\
\text { Prac (2011) }\end{array}$ & \begin{tabular}{l|} 
EPIPC \\
vs. early \\
postoperative \\
intravenous \\
chemotherapy
\end{tabular} & NA. & NA. & $\begin{array}{l}\text { SR: 1-, 2-, 3-, } \\
5-\quad \text { y: favor } \\
\text { EPIPC. Replase } \\
\text { rate: 2-, 3-y } \\
\text { intra-abdominal } \\
\text { recurrence: } \\
\text { favor EPIPC. }\end{array}$ & $\begin{array}{l}\text { Nausea, vomiting: } \\
\text { lower in EPIPC. } \\
\text { Liver and renal } \\
\text { function protection: } \\
\text { favor EPIPC }\end{array}$ & $\begin{array}{l}\text { EPIPC improved } \\
\text { survival rate and } \\
\text { reduced both } \\
\text { recurrence rate and } \\
\text { side effects. }\end{array}$ \\
\hline
\end{tabular}

non Platinum-based regimens [28].

\section{Cisplatin-based therapy vs. non Cisplatin-based therapy}

Only one meta-analysis compared the outcomes of cisplatin-based therapy versus non cisplatin-based therapy. The results demonstrated that cisplatin-free combination therapy significantly enhanced OS, RR and PFS compared with cisplatin-based combination chemotherapy [33].

\section{Targeted therapy}

The efficacy of targeted therapy was evaluated in three meta-analyses $[10,18,22]$. As for OS, one of them favored targeted therapy of antiangiogenic and HER2 pathway but not for EGFR pathway compared with conventional chemotherapy [10]. One showed that in patients with Eastern Cooperative Oncology Group Performance Status (ECOG-PS) of 0, targeted therapy did not report a significant benefit over BSC. Contrarily, chemotherapy was associated with better survival compared to targeted therapy. In patients with ECOG-PS $=1$ or 2 , targeted therapy was superior to BSC in terms of OS. However, it did not show significant difference when comparing to chemotherapy [18]. Another metaanalysis that compared anti-VEGF therapy with non-antiVEGF therapy favored anti-VEGF therapy regarding OS [22]. When it comes to RR, one of them favored antiHER2 agents but not for anti-EGFR and anti-angiogenic agents [10]. One comparing anti-VEGF therapy with non-anti-VEGF therapy favored anti-VEGF therapy in terms of objective RR [22]. One meta-analysis did not reported relevant data. Only one meta-analysis evaluated PFS, which indicated that the targeted therapy of antiangiogenic and HER2 pathway but not EGFR pathway was superior to conventional chemotherapy [10]. Also, only one meta-analysis reported recurrence-free survival (RFS) that anti-VEGF agents reported a significant benefit over non-anti-VEFG agents [22]. As for toxicity, one metaanalysis showed that the incidence of diarrhea and rash was higher in targeted therapy [10]; another found that grade 3 or 4 thrombocytopenia, diarrhea, and hypertension was significantly higher in anti-VEGF therapy compared to non-anti-VEFG therapy [22].

Only RCT studies, rather than non-RCTs were included in the three included meta-analyses.

The meta-analysis by Ciliberto had the largest number of included studies $(n=22)$ followed by the meta-analyses by Qi $(n=7)$ and by Iacovelli $(n=2)$ (Supplementary Table S17). Notably, there was an overlap of included studies between the meta-analyses by Iacovelli and by Ciliberto. All studies about targeted therapy that were included in the meta-analysis by Iacovelli were also covered in the meta-analysis by Ciliberto.

Given its superiority in the quantity of RCT studies, the results of the meta-analysis by Ciliberto might be more reliable. In details, targeted therapy showed a 
Table 18: Findings of meta-analyses: An overview of included studies regarding TCM

\begin{tabular}{|c|c|c|c|c|c|c|}
\hline $\begin{array}{l}\text { First } \\
\text { Author }\end{array}$ & $\begin{array}{l}\text { Journal } \\
\text { (Year) }\end{array}$ & Comparisons & OS & RR & Other & Major comments \\
\hline \multicolumn{7}{|c|}{ SQFZ injection + chemotherapy vs. chemotherapy alone } \\
\hline Li J[11] & $\begin{array}{l}\text { Chin J Integr } \\
\text { Med (2015) }\end{array}$ & $\begin{array}{l}\text { SQFZ injection+ } \\
\text { chemotherapy vs. } \\
\text { chemotherapy } \\
\text { alone }\end{array}$ & NA. & NA. & $\begin{array}{|lr|}\text { Quality rr } & \text { of } \\
\text { life, complete } & \text { comission } \text { and } \\
\text { remion } & \text { partial remission, } \\
\text { AEs: r favor } \\
\text { SQFZ injection+ } \\
\text { chemotherapy. } \\
\end{array}$ & $\begin{array}{l}\text { This systematic } \\
\text { review found } \\
\text { encouraging } \\
\text { albeit limited } \\
\text { evidence for SFI } \\
\text { combined with } \\
\text { chemotherapy. } \\
\end{array}$ \\
\hline Yao K[25] & $\begin{array}{l}\text { J Cancer Res } \\
\text { Ther (2014) }\end{array}$ & $\begin{array}{l}\text { SQFZ injection } \\
+\quad \text { chemotherapy } \\
\text { vs. chemotherapy } \\
\text { alone }\end{array}$ & NA. & $\begin{array}{lr}\text { Overall } & \text { RR: } \\
\text { favor } & \text { SQFZ } \\
\text { +chemotherapy. }\end{array}$ & $\begin{array}{l}\text { The Karnofsky } \\
\text { score (KPS): } \\
\text { higher in SQFZ } \\
\text { injection+ } \\
\text { chemotherapy. }\end{array}$ & $\begin{array}{l}\text { SQFZ+ } \\
\text { chemotherapy } \\
\text { could improve the } \\
\text { clinical efficacy } \\
\text { and performance } \\
\text { status in patients } \\
\text { with AGC. }\end{array}$ \\
\hline \multicolumn{7}{|c|}{ Huachansu+chemotherapy vs. chemotherapy alone } \\
\hline Xie X[37] & $\begin{array}{l}\text { Med } \\
\text { Hypotheses } \\
(2013)\end{array}$ & $\begin{array}{l}\text { Huachansu+ } \\
\text { chemotherapy vs. } \\
\text { chemotherapy } \\
\text { alone }\end{array}$ & $\begin{array}{l}\text { OS: 1-year: } \\
\text { statistically } \\
\text { similar. }\end{array}$ & NA. & $\begin{array}{l}\text { Total RR, KPS, } \\
\text { gastrointestinal } \\
\text { side effects, } \\
\text { leucocytopenia: } \\
\begin{array}{l}\text { favor Huachansu+ } \\
\text { chemotherapy }\end{array}\end{array}$ & $\begin{array}{l}\text { Huchansu+ } \\
\text { chemotherapy } \\
\text { improved } \\
\text { RR, increased } \\
\text { Karnofsky score } \\
\text { and reduced } \\
\text { leucocytopenia. }\end{array}$ \\
\hline \multicolumn{7}{|c|}{ Compound matrine injection+cisplatin chemotherapy vs. cisplatin chemotherapy } \\
\hline $\begin{array}{l}\text { Huang } \\
\mathrm{S}[43]\end{array}$ & 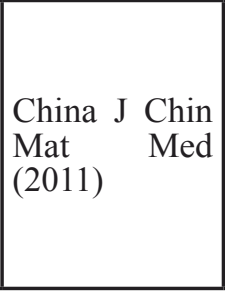 & $\begin{array}{l}\text { Compound matrine } \\
\text { injection+ cisplatin } \\
\text { vs. cisplatin }\end{array}$ & NA. & NA. & $\begin{array}{l}\text { Quality of life, } \\
\text { clinical efficacy, } \\
\text { leukopenia, } \\
\text { thrombocytopenia, } \\
\text { gastrointestinal } \\
\text { AEs: favor } \\
\text { combination } \\
\text { therapy. }\end{array}$ & $\begin{array}{l}\text { Compound matrine } \\
\text { injection+ cisplatin } \\
\text { chemotherapy } \\
\text { could improve the } \\
\text { quality of life with } \\
\text { lower AEs. }\end{array}$ \\
\hline \multicolumn{7}{|c|}{ KLT+chemotherapy vs. chemotherapy alone } \\
\hline $\begin{array}{l}\text { Wang } \\
\mathrm{C}[47]\end{array}$ & $\begin{array}{lr}\text { Mod J } & \text { Integr } \\
\text { Tradi } & \text { Chin } \\
\text { West } & \text { Med } \\
(2011) & \end{array}$ & $\begin{array}{l}\text { KLT+ } \\
\text { chemotherapy vs. } \\
\text { chemotherapy } \\
\text { alone }\end{array}$ & $\begin{array}{l}\text { OS: 1-y: KLT+ } \\
\text { chemotherapy. }\end{array}$ & NA. & 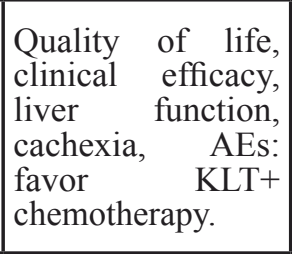 & $\begin{array}{l}\text { KLT+ } \\
\text { chemotherapy } \\
\text { improved the } \\
\text { curative effect and } \\
\text { survival rate with } \\
\text { lower incidence of } \\
\text { AEs. }\end{array}$ \\
\hline
\end{tabular}

*no data was reported regarding SR, DFS, RFS, PFS, Recurrence, TTF, TTP in the included studies

significant survival benefit, which could be ascribed to anti-angiogenic and anti-HER2 agents. Moreover, diarrhea occurrence was higher in anti-HER2 agents while rash occurrence was higher in anti-EGFR drugs.

\section{Combination (doublet/triplet) therapy vs. single/ doublet therapy}

There were four meta-analyses to compare the outcomes of combination therapy, which included doublet and triplet therapy versus monotherapy or doublet therapy $8,39,51,59]$. Three of them came to the conclusion that the combination therapy was superior to single or doublet therapy in terms of OS $[8,39,51]$ while the other one did not reported survival data. Notably, subgroup analyses based on treatment regimens in one of the meta-analyses showed that targeted agent plus cytotoxic chemotherapy significantly improved OS, but not for doublet cytotoxic chemotherapy [8]. As for RR, two of them showed that the combination therapy was favorable over single or doublet therapy [8, 51]. Similarly, subgroup analyses based on treatment regimens in one of the meta-analyses showed that targeted agent plus cytotoxic chemotherapy significantly improved objective RR, but not for doublet cytotoxic chemotherapy [8]. Another meta-analysis favored triplet combination therapy over doublet combination therapy in terms of overall RR [39]. One 
meta-analysis did not reported RR. As for PFS, the only one meta-analysis indicated that doublet combination therapy was associated with better PFS [8]. Besides, only one meta-analysis reported TTP which demonstrated that TTP was significantly improved by the use of combination therapy [51]. As for toxicity, three of them revealed that the toxicity of combination therapy was significantly higher compared to momotherapy or doublet therapy $[8,51,59]$ while the other did not report toxicity in general. However, the incidence of grade 3 or 4 AEs was statistically similar between two groups according to one of the meta-analyses [39].

Only RCT studies, rather than non-RCTs were included in the four meta-analyses.

The meta-analysis by Wagner 2010 had the largest number of included studies $(n=13)$ followed by the metaanalyses by Liu $(n=12)$, Wagner $2006(n=11)$ and Zhang $(n=10)$ (Supplementary Table S18). Notably, there was an overlap of included studies among these four articles.

Given its superiority in the quantity of RCT studies, the results of the meta-analysis by Wagner 2010 might be more reliable. In details, combination chemotherapy improved survival compared to singleagent therapy. However, toxicity was higher in combination chemotherapy. In addition, the meta-analysis of Zhang indicated that no significant survival benefits were observed in doublet cytotoxic chemotherapy regimens while the addition of targeted agent to monochemotherapy as salvage treatment for pretreated AGC patients had substantial survival benefits.

\section{FU/anthracycline-containing regimens with vs. without} cisplatin

Two meta-analyses compared the outcomes of FU/ anthracycline-containing therapy with versus without cisplatin. Both of them favored three-drug combination therapy. Other outcomes were not evaluated between the two groups [51, 59].

Only RCTs studies, rather than non-RCTs were included in the two meta-analyses.

The two meta-analyses shared completely the same included studies $(n=7)$ (Supplementary Table S19). Thus the results were completely consistent with each other. In details, they all agreed that best survival results were achieved with three-drug regimens containing FU, anthracycline, and cisplatin.

\section{FU/cisplatin-containing regimens with vs. without anthracyclines}

Two meta-analyses evaluated the outcomes of FU/cisplatin-containing regimens with $v s$. without anthracyclines. Both of them favor three-drug combination therapy. Other outcomes were not evaluated between the two groups [51, 59].

Only RCT studies, rather than non-RCTs were included in the two meta-analyses.

The number of studies included in these two articles was equal $(n=3)$ (Supplementary Table S20). The included studies were the same between the two metaanalyses and the results were completely consistent with each other. In details, they all agreed that best survival results were achieved with three-drug regimens containing FU, anthracycline, and cisplatin.

\section{Docetaxel-containing vs. non-docetaxel-containing therapy}

One meta-analysis focused on the efficacy of docetaxel-containing regimens. According to the study, Docetaxel containing therapy seemed not to be associated with significant better OS, objective RR and TTP compared to non-docetaxel based therapy. Besides, there were also no significant differences in treatment related death or treatment discontinuation due to toxicity between two groups [51].

\section{Chemotherapy regimens with vs. without lentinan administration}

Lentinan containing chemotherapy was evaluated in one meta-analysis. OS was enhanced by the use of the lentinan combination therapy [56]. Other outcomes were not reported in the study.

\section{EPIPC vs. early postoperative intravenous chemotherapy}

One meta-analysis compared EPIPC with early postoperative intravenous chemotherapy. EPIPC was associated with better 1-, 2-, 3-, and 5-year survival rate compared to early postoperative intravenous chemotherapy. Besides, 2- and 3-year intra-abdominal recurrence was significantly reduced by the use of early postoperative intravenous chemotherapy. Significant lower incidence of nausea and vomiting were observed in EPIPC group. Additionally, EPIPC was superior to intravenous chemotherapy in terms of liver and renal protection [54].

\section{Traditional chinese medicine (TCM)}

\section{Shenqifuzheng (SQFZ) injection plus chemotherapy vs. chemotherapy alone}

Two meta-analyses compared the outcomes of SQFZ injection combined with chemotherapy versus chemotherapy alone $[11,25]$. Only one meta-analysis reported overall RR, which showed that chemotherapy combined with SQFZ injection was favorable over chemotherapy alone [25]. Besides, one of them indicated that significant benefit was observed in SQFZ injection plus chemotherapy in terms of quality of life, complete and partial remission, and AEs compared with chemotherapy alone [11]. The other meta-analysis suggested that the Karnofsky score (KPS) was significantly higher in chemotherapy combined with SQFZ injection [25].

Only RCT studies, rather than non-RCTs were included in the meta-analysis by Li while the information 
regarding RCT studies was not available in the metaanalysis by Yao.

The meta-analysis by Yao had the largest number of included studies $(n=15)$ followed by the meta-analysis by Li $(n=13)$ (Supplementary Table S21). Notably, there was an overlap of included studies between them.

Given its superiority in the quantity of RCT studies, the results of the meta-analysis by Li might be more reliable. In details, SQFZ injection combined with chemotherapy could improve the clinical efficacy and performance status in patients with AGC.

\section{Huachansu plus chemotherapy vs. chemotherapy alone}

Only one meta-analysis evaluated the efficacy of Huachasu plus chemotherapy versus chemotherapy alone. There was no significant difference in 1-year OS between two groups. Besides, Huachasu plus chemotherapy was favorable over chemotherapy alone in term of total RR, KPS, gastrointestinal side effects, and leucocytopenia [37].

\section{Compound matrine injection plus cisplatin therapy vs. cisplatin therapy alone}

One meta-anaysis compared the outcomes between compound matrine injection plus cisplatin regimen versus cisplatin regimen alone. Quality of life and clinical efficacy were favorable for compound matrine injection combined with cisplatin therapy over cisplatin therapy alone. Additionally, compound matrine injection plus cisplatin therapy was associated with significantly lower incidence of leukopenia, thrombocytopenia, and gaastrointestinal AEs compared to cisplatin therapy alone [43].

\section{Kanglaite (KLT) plus chemotherapy vs. chemotherapy alone}

One meta-analysis evaluated the efficacy of KLT combined with chemotherapy regimen. KLT significantly improved 1-year OS compared to chemotherapy alone. KLT plus chemotherapy was associated with better quality of life and clinical efficacy compared with chemotherapy alone according to the results. Besides, KLT combined with chemotherapy regimen was superior to chemotherapy alone in terms of liver protection, incidence of cachexia and AEs [47].

\section{DISCUSSIONS}

The treatment for AGC include surgery gasterectomy, perioperative chemotherapy with or without chemoradiation, palliative chemotherapy, targeted therapy $\mathrm{BSC}$ and other.

Surgery remains the most important component of curative therapy [61]. According to the JGCA treatment guidelines, distal gastrectomy with D2 lymphadenectomy via open approach is the standard procedure for AGC [5]. It was suggested in our study that LG was a safe and technical alternative to $\mathrm{OG}$ for AGC patients with a lower complication rate and enhanced postoperative recovery. The results were consistent with recent multicenter RCT and research $[62,63]$. Besides, NAC and AC were thought to benefit the survival over surgery alone though no clear superiority of one strategy over another has emerged [41, 57]. In metastatic setting, chemotherapy is the mainstay treatment. And it was demonstrated in the present study that targeted therapy like anti-angiogenic and anti-HER2 agents but anti-EGFR agents might have a significant survival benefit [10]. Notably, it was suggested that addition of TCM such as SQFZ injection to chemotherapy could improve clinical efficacy and benefit the quality of life compared to chemotherapy alone $[11,37]$.

\section{Limitations}

We recognize that the present review has some limitations. First, not all the related survival and clinical data were evaluated in every meta-analysis. As a result, made it difficult to compare directly between metaanalyses. Second, the accuracy of the findings in every meta-analysis could not be guaranteed since it was not possible for us to repeat every meta-analysis and the heterogeneity among included studies in every metaanalysis were not considered. Third, there was overlap of the original studies in each meta-analysis; and we arbitrarily evaluated the reliability of meta-analysis according to the quality and quantity of the included metaanalysis. Fourth, conversion therapy, immune therapy and radiation therapy were not discussed in the present study.

\section{Recommendations}

\section{Surgery}

1. $\mathrm{LG}$ is a safe and technical alternative to $\mathrm{OG}$ for AGC patients with a lower complication rate and enhanced postoperative recovery (grade of recommendation: moderate).

2. NAC can improve the tumor resection rate and the survival rate in AGC patients without increasing the operative risk and perioperative mortality (grade of recommendation: high).

3. Postoperative chemotherapy can improve OS after radical surgery for gastric cancer (grade of recommendation: high).

4. IPC has positive effect on overall and peritoneal recurrence and distant metastasis. Besides, loco-regional lymph-nodes invasion in patients affected by AGC is not a contraindication to IPC (grade of recommendation: high).

5. Palliative gastrectomy for patients with incurable AGC was associated with longer OS, especially for patients with stage M1 gastric cancer (grade of recommendation: low). 
6. Palliative gastrectomy combined with hepatectomy might provide better OS than palliative gastrectomy only (grade of recommendation: low).

7. Avoiding the use of abdominal drains might reduce drain-related complications and shortened hospital stay after gastrectomy (grade of recommendation: high).

\section{Chemotherapy}

1. Chemotherapy significantly improved survival in comparison to $\mathrm{BSC}$ (grade of recommendation: high).

2. S-1-based regimens are effective and tolerable as first-line treatment of AGC in both Asian and Western countries though the toxicity profile of S-1 was clearly more advantageous in Western patients (grade of recommendation: high).

3. S-1 and capecitabine could be used interchangeably for AGC, at least in Asia (grade of recommendation: high).

4. S-1 combination therapy was more efficacious than S-1 monotherapy though AEs was higher and safety profile was poorer in S-1 combination therapy (grade of recommendation: high).

5. Oxaliplatin-based therapy was associated with a small but significant survival benefit with less toxicity and better tolerability (grade of recommendation: moderate).

6. CPT-11-containing regimen had survival benefit as first-line treatment for AGC patients (grade of recommendation: high).

7. New-generation agent such as S-1, taxanes and irinotecan seemed to be valid options for patients with inoperable AGC as first-line chemotherapy (grade of recommendation: high).

8. Replacing cisplatin with oxaliplatin, CPT-11, or taxane significantly enhanced OS, RR and PFS compared with cisplatin-based combination chemotherapy (grade of recommendation: high).

9. Targeted therapy may have a significant survival benefit, which could be ascribed to anti-angiogenic and anti-HER2 agents (grade of recommendation: high).

10. Combination chemotherapy may improve survival compared to single-agent therapy at a price of higher toxicity (grade of recommendation: high).

11. Better survival results should be achieved in FU, anthracycline and cisplatin combination therapy no matter comparing to FU/anthracycline doublet regimens FU/or FU/cisplatin doublet regimens (grade of recommendation: high).

12. The addition of lentinan to standard chemotherapy may offer a significant advantage over chemotherapy alone in terms of survival (grade of recommendation: high).

13. EPIPC may improve survival rate and reduce both recurrence rate and side effects (grade of recommendation: high).

\section{TCM}

1. SQFZ injection combined with chemotherapy could improve the clinical efficacy and performance status in patients with AGC (grade of recommendation: high).

2. Huchansu combined with chemotherapy provides benefits for AGC on improving the response rate, increasing Karnofsky score, reducing leucocytopenia and major side effects (grade of recommendation: high).

3. Compound matrine injection combined with cisplatin chemotherapy can improve the quality of life with lower AEs (grade of recommendation: high).

\section{Uncertainties}

1. D4 lymphadenectomy should be performed prudently for its wound degree of surgery is significantly higher. Standard operating procedures should be established to reduce wound degree.

2. The evidence is limited to confirm that XELOX may share similar efficacy as FOLFOXs and reduce toxicities.

3. Though the addition of docetaxel to the cisplatin/fluorouracil combination may provide survival benefit, the clinical value of this regimen is regarded as controversial for its significant toxicity.

4. The benefit of KLT plus chemotherapy may be confirmed in further rigorously controlled trials.

\section{MATERIALS AND METHODS}

\section{Search strategy and study selection}

All meta-analysis papers regarding the treatment of AGC via the PubMed, Google Scholar, and Web of Science were retrieved. The search strategy terms used in the English databases were "treatment OR management OR therapy" AND "advanced gastric cancer OR advanced gastric carcinoma OR advanced gastric neoplasm OR advanced stomach cancer OR advanced stomach carcinoma OR advanced stomach neoplasm" AND "metaanalyses". The last search was performed on March, 13, 2016.

Eligibility criteria were as follows. 1) All metaanalyses regarding the treatment of AGC. 2) Duplicate 
publications. 3) Only abstract available. 4) Meeting abstracts. 5) Only systematic reviews without metaanalyses. 6) Patients without AGC. 7) Other topics, but not treatment modalities.

Primary outcomes were OS, RR, DFS, RFS, PFS TTP, TTF and other endpoints.

\section{Reliability of meta-analyses}

As the results were different among the metaanalyses, the reliability was evaluated according to the quality and quantity of original studies included in every meta-analysis. First, the results of a meta-analysis would be more reliable if a larger number of RCTs were included. And if the larger number of patients were included in the RCTs, the results of a meta-analysis would be more reliable. Second, the number of non-RCT studies was further evaluated if the number of RCTs and patients were similar. Third, if the number of RCTs and non-RCT studies included was similar but the results were not consistent among meta-analyses, the total number of the included patients and statistical methods would be further evaluated. If the hazard ratio was calculated, the results of a meta-analysis would be more reliable.

\section{Grade of recommendations}

Grade of recommendation was determined in the way that was suggested by a previous study [64]. High grade recommendation was considered if the results of meta-analyses were based on more than 3 single-center RCTs or 1 multi-center RCT. Low grade recommendation was considered, if the results of meta-analyses were based on the non-RCT studies alone. As for something in between, moderate grade recommendation was considered.

\section{Abbreviations}

AGC, advanced gastric cancer; LG, laparoscopic gastrectomy; OG, open gastrectomy; $\mathrm{BSC}$, basic supportive care; RCT, randomized controlled trial; NAC, neoadjuvant chemotherapy; AC, adjuvant chemotherapy; IPC, intraperitoneal chemotherapy; HIIPC, hyperthermic intraoperative intraperitoneal chemotherapy; EPIPC, early postoperative intraperitoneal chemotherapy; NIIPC, normothermic intraoperative intraperitoneal chemotherapy; DPIPC delayed postoperative intraperitoneal chemotherapy; CPT-11, irinotecan; TCM, Traditional Chinese Medicine; SQFZ, Shenqifuzheng; KLT, Kanglaite; OS, overall survival; RR, response rate; DFS, disease-free survival; RFS, recurrence-free survival; PFS, progression-free survival; TTP, time-to-progression; $\mathrm{TTF}$, time-to failure.

\section{ACKNOWLEDGMENTS}

The authors have declared no conflicts of interest. This research did not receive any specific grant from funding agencies in the public, commercial, or not-forprofit sectors.

\section{CONFLICTS OF INTEREST}

The authors have declared no conflicts of interest

\section{Author Contributions}

Study concepts and design: Qi X, Li G, Zhao Y; Literature search: Wang W, Cai D; Data extraction: Liu Y, Li W, Li F; Manuscript preparation and revision: Liu Y, Qi X, Wang W. All authors have participated sufficiently in the study and approved the final version.

\section{FINANCIAL SUPPORT}

This work was supported by the grants from National Natural Science Foundation of China (81600510; 81672446; 814021797), Natural Science Foundation of Guangdong Province (201508030042), Major Program of Collaborative Innovation of Guangzhou (2015A030313089) and Science and Technology Project of Guangdong Province (2015A040404026; 2016A040403064).

\section{REFERENCES}

1. Global Burden of Disease Cancer Collabration, Fitzmaurice C, Dicker D, Pain A, Hamavid H, Moradi-Lakeh M, Maclntyre MF, Allen C, Hansen G, Woodbrook R, Wolfe C, Hamadeh RR, Moore A, et al. The Global Burden of Cancer 2013. JAMA Oncol 2015; 1: 505-527.-

2. World Health Organisation, Stomach Cancer: Estimated Incidence, Mortality and Prevalence Worldwide in 2012, 2012. http://globocan.iarc.fr/old/FactSheets/cancers/ stomach-new.asp.

3. Chen W, Zheng R, Baade P D, Zhang S, Zeng H, Bray F, Jemal A, Yu X, PhD, He J. Cancer statistics in China, 2015. CA: a cancer journal for clinicians, 2016; 66: 115-132.

4. Orditura M. Treatment of gastric cancer. World Journal of Gastroenterology 2014; 20:1635.

5. Japanese Gastric Cancer Association. Japanese gastric cancer treatment guidelines 2010 (ver. 3). Gastric Cancer 2011; 14: 113-123.

6. Antonia Digklia, Anna Dorothea Wagner. Advanced gastric cancer: Current treatment landscape and future perspectives. World J Gastroenterol 2016; 22: 2403-2414.

7. Ter Veer E, Mohammad NH, Lodder P, Ngai LL, Samaan M, van Oijen MG, van Laarhoven HW. The efficacy and 
safety of S-1-based regimens in the first-line treatment of advanced gastric cancer: a systematic review and metaanalysis. Gastric Cancer. 2016.

8. Zhang Y, Ma B, Huang XT, Li YS, Wang Y, Liu ZL. Doublet Versus Single Agent as Second-Line Treatment for Advanced Gastric Cancer: A Meta-Analysis of 10 Randomized Controlled Trials. Medicine (Baltimore). 2016; 95: e2792.

9. Badiani B, Maratea D, Messori A. Second-line treatments for advanced gastric cancer: Interpreting outcomes by network meta-analysis. World J Clin Oncol. 2015; 6: 73-79.

10. Ciliberto D, Staropoli N, Caglioti F, Gualtieria S, Fiorilloa L, Chiellinoa S, Angelisa A M D, Mendicinoa F, Bottaa C, Caragliabc M, Tassoneac P, Tagliaferria P. A systematic review and meta-analysis of randomized trials on the role of targeted therapy in the management of advanced gastric cancer: Evidence does not translate? Cancer Biol Ther. $2015 ; 16: 1148-1159$.

11. Li J, Wang JC, Ma B, Gao W, Chen P, Sun R, Yang KH. Shenqi Fuzheng Injection for advanced gastric cancer: a systematic review of randomized controlled trials. Chin J Integr Med. 2015; 21: 71-79.

12. Lu C, Zhou S, Peng Z, Chen L. Quality of D2 lymphadenectomy for advanced gastric cancer: is laparoscopic-assisted distal gastrectomy as effective as open distal gastrectomy? Surg Endosc. 2015; 29: 1537-1544.

13. Quan Y, Huang A, Ye M, Xu M, Zhuang B, Zhang P, Yu $\mathrm{B}$ and Min Z. Comparison of laparoscopic versus open gastrectomy for advanced gastric cancer: an updated metaanalysis. Gastric Cancer. 2015.

14. Wu FL, Lu DC, Ying YP, Huang JJ, Zhou AM, Jiang DK, Chen MW, Yang X, Zhou J, Huang HQ, Zeng HY. A Meta-analysis Reveals S-1-based Chemotherapy Improves the Survival of Patients With Advanced Gastric Cancer. Medicine (Baltimore). 2015; 94: e652.

15. Xu HB, Huang F, Su R, Shen FM, Lv QZ. Capecitabine plus oxaliplatin (XELOX) compared with 5-fluorouracil/ leucovorin plus oxaliplatin (FOLFOXs) in advanced gastric cancer: meta-analysis of randomized controlled trials. Eur J Clin Pharmacol. 2015; 71: 589-601.

16. Coccolini F, Cotte E, Glehen O, Lotti M, Poiasina E, Catena F, Yonemura Y, Ansaloni L. Intraperitoneal chemotherapy in advanced gastric cancer. Meta-analysis of randomized trials. Eur J Surg Oncol (EJSO). 2014; 40: 12-26.

17. Huang YL, Lin HG, Yang JW, Jiang FQ, Zhang T, Yang HM, Li CL, Cui Y. Laparoscopy-assisted versus open gastrectomy with D2 lymph node dissection for advanced gastric cancer: a meta-analysis. Int J Clin Exp Med. 2014; 7: 1490-1499.

18. Iacovelli R, Pietrantonio F, Farcomeni A, Maggi C, Palazzo A, Ricchini F, de Braud F, Di Bartolomeo M. Chemotherapy or targeted therapy as second-line treatment of advanced gastric cancer. A systematic review and meta-analysis of published studies. PLOS ONE. 2014; 9: e108940.

19. Li DH, Pan ZK, Ye F, An HX, Wu JX. S-1-based versus 5-FU-based chemotherapy as first-line treatment in advanced gastric cancer: a meta-analysis of randomized controlled trials. Tumour Biol. 2014; 35: 8201-8208.

20. Liu GF, Tang D, Li P, Wang S, Xu YX, Long AH, Zhou NL, Zhang LL, Chen J, Xiang XX. S-1-based combination therapy vs S-1 monotherapy in advanced gastric cancer: a meta-analysis. World J Gastroenterol. 2014; 20: 310-318.

21. Liu H, Chen X, Sun J, Gao P, Song Y, Zhang N, Lu X, Xu $\mathrm{H}$, Wang $\mathrm{Z}$. The efficacy and toxicity of paclitaxel plus S-1 compared with paclitaxel plus 5-FU for advanced gastric cancer: a PRISMA systematic review and meta-analysis of randomized controlled trials. Medicine (Baltimore). 2014; 93: e164.

22. Qi WX, Shen Z, Tang LN, Yao Y. The role of anti-VEGF agents in the treatment of advanced gastric cancer: a metaanalysis of randomized controlled trials. Tumour Biol. 2014; 35: 7675-7683.

23. Wu JR, Tang WZ, Chen X, Xie YT, Chen SY, Peng QL, Xie L, Deng Y, Li TJ, He Y, Wang J, Li S, Qin X. S-1based therapy versus $\mathrm{S}-1$ monotherapy in advanced gastric cancer: a meta-analysis. Tumour Biol. 2014; 35: 3283-3293.

24. Yang J, Zhou Y, Min K, Yao Q, Xu CN. S-1-based vs non-S-1-based chemotherapy in advanced gastric cancer: a meta-analysis. World J Gastroenterol. 2014; 20: 1188611893.

25. Yao K, Ma Y, Ma W, Hu J, Wang C, Chen J, Zhang J, Hua L, Ren X. Shenqifuzheng injection combined with chemotherapy in the treatment of advanced gastric cancer: a systematic review and meta-analysis. J Cancer Rea Ther. 2014; 10 Suppl 1: 70-74.

26. Zou ZH, Zhao LY, Mou TY, Hu YF, Yu J, Liu H, Chen $\mathrm{H}$, Wu JM, An SL, Li GX. Laparoscopic vs open D2 gastrectomy for locally advanced gastric cancer: a metaanalysis. World J Gastroenterol. 2014; 20: 16750-16764.

27. Chen K, Xu XW, Mou YP, Pan Y, Zhou YC, Zhang RC, $\mathrm{Wu}$ D. Systematic review and meta-analysis of laparoscopic and open gastrectomy for advanced gastric cancer. World $\mathrm{J}$ Surg Oncol. 2013; 11: 182.

28. Chen WW, Wang F, Xu RH. Platinum-based versus non-platinum-based chemotherapy as first line treatment of inoperable, advanced gastric adenocarcinoma: a metaanalysis. PLOS ONE. 2013; 8: e68974.

29. Choi YY, Bae JM, An JY, Hyung WJ, Noh SH. Laparoscopic gastrectomy for advanced gastric cancer: are the long-term results comparable with conventional open gastrectomy? A systematic review and meta-analysis. J Surg Oncol. 2013; 108: 550-556.

30. He MM, Wu WJ, Wang F, Wang ZQ, Zhang DS, Luo HY, Qiu MZ, Wang FH, Ren C, Zeng ZL, Xu RH. S-1-based chemotherapy versus capecitabine-based chemotherapy as first-line treatment for advanced gastric carcinoma: a metaanalysis. PLOS ONE. 2013; 8: e82798. 
31. Kim HS, Kim HJ, Kim SY, Kim TY, Lee KW, Baek SK, Kim TY, Ryu MH, Nam BH, Zang DY. Secondline chemotherapy versus supportive cancer treatment in advanced gastric cancer: a meta-analysis. Ann Oncol. 2013; 24: 2850-2854.

32. Mi DH, Li Z, Yang KH, Cao N, Lethaby A, Tian JH, Santesso N, Ma B, Chen YL, Liu YL. Surgery combined with intraoperative hyperthermic intraperitoneal chemotherapy (IHIC) for gastric cancer: a systematic review and meta-analysis of randomised controlled trials. Int J Hyperthermia. 2013; 29: 156-167.

33. Petrelli F, Zaniboni A, Coinu A, Cabiddu M, Ghilardi M, Sgroi G, Barni S. Cisplatin or not in advanced gastric cancer: a systematic review and meta-analysis. PLOS ONE. 2013; 8:e83022.

34. Qiu J, Pankaj P, Jiang H, Zeng Y, Wu H. Laparoscopy versus open distal gastrectomy for advanced gastric cancer: a systematic review and meta-analysis. Surg Laparosc Endosc Percutan Tech. 2013; 23: 1-7.

35. Qi WX, Shen Z, Lin F, Sun YJ, Min DL, Tang LN, He AN, Yao Y. Overall survival benefits for irinotecan-containing regimens as first-line treatment for advanced gastric cancer: an updated meta-analysis of ten randomized controlled trials. Int J Cancer. 2013; 132: 66-73.

36. Sun J, Song Y, Wang Z, Chen X, Gao P, Xu Y, Zhou B, $\mathrm{Xu} \mathrm{H}$. Clinical significance of palliative gastrectomy on the survival of patients with incurable advanced gastric cancer: a systematic review and meta-analysis. BMC Cancer. 2013; 13: 577 .

37. Xie X, Huang X, Li J, Lv X, Huang J, Tang S, Sun Y. Efficacy and safety of Huachansu combined with chemotherapy in advanced gastric cancer: a meta-analysis. Med Hypotheses. 2013; 81: 243-250.

38. Ye LY, Liu DR, Li C, Li XW, Huang LN, Ye S, Zheng YX, Chen L. Systematic review of laparoscopy-assisted versus open gastrectomy for advanced gastric cancer. J Zhejiang Univ Sci B. 2013; 14: 468-478.

39. Liu N, Lu J, Ding X. Effectiveness and safety of triplet combination chemotherapy compared to doublet combination chemotherapy for advanced gastric cancer: a meta-analysis. Chinese Journal of Hospital Pharmacy. 2012; 32: 1467-1472.

40. Ma Y, Tang L, Wang HX, Xu YC, Ma Y, Zhang FC. Capecitabine for the treatment for advanced gastric cancer: efficacy, safety and ethnicity. J Clin Pharm Ther. 2012; 37: 266-275.

41. Feng X, Bin L, Hongying G, Jiyun W, Qingxian Z. A Metaanalysis on curative effects of neoadjuvant chemotherapy for treating advanced gastric cancer. Chongqing Medicine. 2012; 41: 2591-2594.

42. Huang J, Cao Y, Wu L, Liao C, He Y, Gao F. S-1-based therapy versus 5-FU-based therapy in advanced gastric cancer: a meta-analysis. Med Oncol. 2011; 28: 1004-1011.

43. Huang S, Fan W, Liu P, Tian J. Meta-analysis of compound matrine injection combined with cisplatin chemotherapy for advanced gastric cancer. Zhongguo Zhong Yao Za Zhi. 2011; 36: 3198-3202.

44. Liu HP, Zhang YC, Zhang YL, Yin LN, Wang J. Drain versus no-drain after gastrectomy for patients with advanced gastric cancer: systematic review and metaanalysis. Dig Surg. 2011; 28: 178-189.

45. Martinez-Ramos D, Miralles-Tena JM, Cuesta MA, Escrig-Sos J, Van der Peet D, Hoashi JS, Salvador-Sanchis JL. Laparoscopy versus open surgery for advanced and resectable gastric cancer: a meta-analysis. Rev Esp Enferm Dig. 2011; 103: 133-141.

46. Montagnani F, Turrisi G, Marinozzi C, Aliberti C, Fiorentini G. Effectiveness and safety of oxaliplatin compared to cisplatin for advanced, unresectable gastric cancer: a systematic review and meta-analysis. Gastric Cancer. 2011; 14: 50-55.

47. Can W, Quan W, Xiran H, Wei P, Quanlin G. Meta-analysis on clinical curative effect of KLT plus Chemotherapy in treatment of advanced gastric cancer. Modern Journal of Integrated Traditional Chinese and Western Medicine. 2011; 20: 3774-3777.

48. Zhang L, Zhang Y, Wu J. Efficacy and Safety of D3 Lymph Node Dissection in Patients with Advanced Gastric Cancer: A Meta-analysis. Chinese General Practice. 2011; 14(6C): 2004-2007.

49. Zhang Y, Wang Q, Wang C. Impact of Early Postoperative Intraperitoneal Chemotherapy on Clinical Survival and Recurrence Rates in Treatment of Advanced Gastric Cancer: A Meta Analysis. Chinese General Practice. 2011; 14(10C): 3487-3490.

50. Li W, Qin J, Sun Y, Liu T. Neoadjuvant chemotherapy for advanced gastric cancer: A meta-analysis. World J Gastroenterol. 2010; 16: 5621-5628.

51. Wagner AD, Unverzagt S, Grothe W, Kleber G, Grothey A, Haerting J, Fleig WE. Chemotherapy for advanced gastric cancer. Cochrane Database Syst Rev. 2010; 3: CD004064.

52. Wang DL, Gu DY, Huang HY, Xu Z, Chen JF. Irinotecaninvolved regimens for advanced gastric cancer: a pooledanalysis of clinical trials. World J Gastroenterol. 2010; 16: 5889-5894.

53. Wang Z, Chen J, Cao Y. Systematic review of D2 lymphadenectomy versus D2 with para-aortic nodal dissection for advanced gastric cancer. WORLD J GASTROENTERO. 2010; 16: 1138-1149.

54. Zhang Y, Shi W, Pan H, Wu J, Zhang Y. A meta-analysis of $\mathrm{D} 2+$ paraaortic lymph node dissection for patients with advanced gastric cancer. Chinese Journal of General Surgery. 2010; 19: 1085-1090.

55. Gong JF, Shen L. Meta-analyses of oxaliplatin-based chemotherapy versus cisplatin-based chemotherapy in advanced gastric cancer. Zhonghua Yi Xue Za Zhi. 2009; 89: 3261-3265. 
56. Oba K, Kobayashi M, Matsui T, Kodera Y, Sakamoto J. Individual patient based meta-analysis of lentinan for unresectable/recurrent gastric cancer. Anticancer Res. 2009; 29: 2739-2745.

57. Sun P, Xiang JB, Chen ZY. Meta-analysis of adjuvant chemotherapy after radical surgery for advanced gastric cancer. Brit J Surg. 2009; 96: 26-33.

58. Yan TD, Black D, Sugarbaker PH, Zhu J, Yonemura Y, Petrou G, Morris DL. A systematic review and metaanalysis of the randomized controlled trials on adjuvant intraperitoneal chemotherapy for resectable gastric cancer. Ann Surg Oncol. 2007; 14: 2702-2713.

59. Wagner AD, Grothe W, Haerting J, Kleber G, Grothey A, Fleig WE. Chemotherapy in advanced gastric cancer: a systematic review and meta-analysis based on aggregate data. J Clin Oncol. 2006; 24: 2903-2909.

60. Casaretto L, Sousa PL, Mari JJ. Chemotherapy versus support cancer treatment in advanced gastric cancer: a meta-analysis. Braz J Med Biol Res. 2006; 39: 431-440.

61. Huang J, Zhao Y, Xu Y, Zhu Y, Huang J, Liu Y, Zhao L, Li Z, Liu H, Wang QL, Qi X. Comparative effectiveness and safety between oxaliplatin-based and cisplatin-based therapy in advanced gastric cancer: a meta-analysis of randomized controlled trials. Oncotarget. 2016; 7:3482431. doi: 10.18632/oncotarget.9189.
62. Hu Y, Huang C, Sun Y, Su X, Cao H, Hu J, Xue Y, Suo J, Tao K, He X, Wei H, Ying M, Hu W, et al. Morbidity and Mortality of Laparoscopic Versus Open D2 Distal Gastrectomy for Advanced Gastric Cancer: A Randomized Controlled Trial. J Clin Oncol. 2016: 34: 1350-1357.

63. Geng X, Liu H, Lin T, Hu Y, Chen H, Zhao L, Mou T, Qi $\mathrm{X}, \mathrm{Yu}$ J, Li G. Survival benefit of gastrectomy for gastric cancer with peritoneal carcinomatosis: a propensity scorematched analysis. Cancer Med 2016 Aug;

64. Qi X, Zhao Y, Li H, Guo X, Han G. Management of hepatocellular carcinoma: An overview of major findings from meta-analyses. Oncotarget. 2016; 7:34703-51. doi: 10.18632/oncotarget.9157. 